\title{
Full-waveform inversion in acoustic orthorhombic media and application to a North Sea data set
}

\author{
Nabil Masmoudi ${ }^{1}$ and Tariq Alkhalifah ${ }^{1}$
}

\begin{abstract}
Full-waveform inversion (FWI) in anisotropic media is challenging, mainly because of the large computational cost, especially in $3 \mathrm{D}$, and the potential trade-offs between the model parameters needed to describe such media. By analyzing the trade-offs and understanding the resolution limits of the inversion, we can constrain FWI to focus on the main parameters the data are sensitive to and push the inversion toward more reliable models of the subsurface. Orthorhombic anisotropy is one of the most practical approximations of the earth subsurface that takes into account the natural horizontal layering and the vertical fracture network. We investigate the feasibility of a multiparameter FWI for an acoustic orthorhombic model described by six parameters. We rely on a suitable parameterization based on the horizontal velocity and five dimensionless anisotropy
\end{abstract}

parameters. This particular parameterization allows a multistage model inversion strategy in which the isotropic, then, the vertical transverse isotropic, and finally the orthorhombic model can be successively updated. We applied our acoustic orthorhombic inversion on the SEG-EAGE overthrust synthetic model. The observed data used in the inversion are obtained from an elastic variable density version of the model. The quality of the inverted model suggests that we may recover only four parameters, with different resolution scales depending on the scattering potential of these parameters. Therefore, these results give useful insights on the expected resolution of the inverted parameters and the potential constraints that could be applied to an orthorhombic model inversion. We determine the efficiency of the inversion approach on real data from the North Sea. The inverted model is in agreement with the geologic structures and well-log information.

\section{INTRODUCTION}

The objective of full-waveform inversion (FWI) is to recover a high-resolution model that is capable of matching the observed seismic data, trace by trace, through repetitive modeling and a local optimization technique (Lailly, 1983; Tarantola, 1984; Virieux and Operto, 2009). Over the past three decades, most FWI techniques have been designed to recover only a P-wave velocity model because of the high computational cost (e.g., Gauthier et al., 1986; Pratt, 1999). The recent progress in high-performance computing and the improvement in data acquisition resulted in the extension of FWI to 2D and 3D acoustic and elastic transverse isotropic media with a vertical axis of symmetry (VTI) (e.g., Warner et al., 2013; Operto et al., 2014; Wu and Alkhalifah, 2016; Djebbi and Alkhalifah, 2017; Kamath et al., 2017). This extension has resulted in challenges related to the trade-offs between the model parameters in our inversion process. The trade-offs between the model parameters and their resolution limits have motivated several studies investigating suitable parameterizations for our inversion schemes (Gholami et al., 2013; Operto et al., 2013; Alkhalifah and Plessix, 2014; Alkhalifah, 2016; Kamath and Tsvankin, 2016; Djebbi et al., 2017; Kazei and Alkhalifah, 2018). Most of these studies rely on the analysis of the radiation (scattering) patterns based on the Born approximation (Wu and Aki, 1985; Panning et al., 2009).

Orthorhombic anisotropy is usually regarded as the most practical realistic approximation of the earth subsurface because it combines the anisotropy admitted by the natural, mostly horizontal layering of the earth (due to gravity), as well as the vertical aligned fractures, usually found in fractured reservoirs or horizontal stresses caused by the tectonic regime or salt intrusions (Cheadle et al.,

\footnotetext{
Manuscript received by the Editor 8 November 2017; revised manuscript received 6 February 2018; published ahead of production 11 June 2018 ; published online 18 July 2018.

${ }^{1}$ King Abdullah University of Science and Technology (KAUST), Physical Science and Engineering Division, Thuwal, Saudi Arabia. E-mail: nabil .masmoudi@kaust.edu.sa; tariq.alkhalifah@kaust.edu.sa.

(C) 2018 Society of Exploration Geophysicists. All rights reserved.
} 
1991; Wild and Crampin, 1991; Schoenberg and Helbig, 1997; Tsvankin, 1997; Bakulin et al., 2000). Contrary to the VTI case, orthorhombic media incorporate velocity variations with azimuths (azimuthal anisotropy), therefore, 3D wide-azimuth acquisitions are essential to estimate these variations (Tsvankin and Grechka, 2011). Azimuthal anisotropy can be described using the fracture weaknesses (Schoenberg and Sayers, 1995; Bakulin et al., 2000) or the deviations anisotropy parameters (Masmoudi et al., 2016).

Practical parameterizations for acoustic and elastic orthorhombic media have been investigated. In the acoustic case, Masmoudi and Alkhalifah (2016a) analytically analyze the radiation patterns of the anisotropic parameter perturbation and end up recommending different parameterizations for different FWI strategies, for instance, those using an initial model obtained from migration velocity analysis (MVA) and those obtained from inverting the diving wave energy. For VTI media, Alkhalifah and Plessix (2014) and Alkhalifah (2016) show that a combination of the horizontal velocity $v_{h}$ and the parameters $\epsilon$ and $\eta$ is optimal for an inversion that starts with the diving wave energy. In this parameterization, diving waves uniquely constrain the long-wavelength variations of the velocity model, $\epsilon$ is mainly sensitive to the model reflectivity, and $\eta$ influences intermediate incidence angles. Furthermore, Guitton and Alkhalifah (2016) show that $\epsilon$ could absorb the density effect because these two parameters have similar scattering properties. Using these insights, Masmoudi and Alkhalifah (2016a) propose an acoustic orthorhombic parameterization based on the three VTI parameters $\left(v_{h 1}, \epsilon_{1}, \eta_{1}\right)$ defined in the $(x, z)$ vertical plane and three additional azimuthal anisotropy parameters: $\epsilon_{d}$ and $\eta_{d}$ describing the deviation in anisotropy between the $(x, z)$ and $(y, z)$ vertical planes and the parameter $\delta_{3}$ describing the anellipticity in the horizontal $(x, y)$ plane. As shown in Masmoudi and Alkhalifah (2016a), such parameterization preserves the attractive features of the three VTI parameters and maintains their scattering potential stationary as a function of azimuth. In the elastic case, Oh and Alkhalifah (2016b) propose an extension of this acoustic orthorhombic parameterization by adding three additional parameters: the vertical S-wave velocity $v_{s 1}$ polarized in the $(x, z)$ plane, the parameter $\gamma_{1}$ in the same plane, and a deviation parameter $\gamma_{d}=\left(\gamma_{2}-\gamma_{1}\right) /\left(1+2 \gamma_{1}\right)$, where $\gamma_{2}$ is defined in the $(y, z)$ plane. Recently, some applications of waveform inversion in elastic and acoustic orthorhombic media have been proposed (Albertin et al., 2016; Alkhalifah et al., 2016; Oh and Alkhalifah, 2016a; Wang and Tsvankin, 2016; Zhang et al., 2017).

The efficiency of wave simulators is a vital ingredient in FWI. Several techniques have been proposed to model $\mathrm{P}$-wave propagation in orthorhombic media, among them solving coupled systems of second-order partial differential equations (Fowler and King, 2011; Cheng and Kang, 2014) and the mixed-domain wavefield extrapolators (Fowler and Lapilli, 2012; Fomel et al., 2013). Alkhalifah (2000) proposes a pseudoacoustic wave equation, which provides accurate simulation of the kinematics of $\mathrm{P}$-wave propagation. $\mathrm{Xu}$ and Zhou (2014) suggest solving this pseudoacoustic VTI wave equation by decomposing the pseudodifferential operator into a scalar operator and a differential operator. The scalar operator involves an asymptotic approximation on calculating the wavefield propagation direction, which resulted in aliasing of the wavefield and dispersion artifacts in finite-difference implementations. To enhance the accuracy of this approach, Xu et al. (2015) propose an elliptic decomposition of the differential operator, which alleviates the magnitude of the scalar operator and provides accurate wave- field solutions. Contrary to solving coupled-system equations, $\mathrm{Xu}$ and Zhou (2014) approach yields a wavefield solution free of $\mathrm{S}$-wave artifacts. Furthermore, this method has been applied in a VTI migration (Mu et al., 2015) and inversion (Le et al., 2015).

In this paper, we focus on the feasibility of inverting the six acoustic orthorhombic model parameters proposed in Masmoudi and Alkhalifah (2016a). Through an inversion of the SEG-EAGE overthrust model, we assess the performance of this parameterization and discuss the resolution limits and the crosstalk between the parameters. The observed data in this inversion correspond to an elastic variable density version of the model. Therefore, we discuss the potential leakage of the elastic and density effects into the acoustic parameters. Furthermore, we apply our inversion to a 3D ocean bottom cable data set from the North Sea. We use total variation (TV) regularization and structural model constraints to resolve sharp interfaces and to push the inversion toward geologically consistent structures. To solve the pseudoacoustic orthorhombic wave equation, we use a similar approach to Xu and Zhou (2014) method. Here, the pseudodifferential operator is decomposed into two operators. The ellipsoid decomposition is used as well to enhance the accuracy. Furthermore, we formulate the adjoint problem and propose the gradients with respect to the model parameters.

We will start by reviewing the parameterization used throughout the paper and discuss its corresponding radiation patterns. Then, we will describe the forward and adjoint problems and discuss our FWI implementation. Finally, we will show applications of our acoustic orthorhombic inversion on a modified SEG-EAGE overthrust model and on 3D real data from the North Sea.

\section{A SUITABle CHOICE OF PARAMETERS}

By analyzing the radiation patterns of the scattering of different orthorhombic parameterizations, Masmoudi and Alkhalifah (2016a) suggest a new parameterization for acoustic orthorhombic media with potentially interesting features for FWI and MVA. Here, we focus on the parameterization, which includes three VTI parameters: the horizontal velocity $v_{h 1}, \epsilon_{1}, \eta_{1}$, which are defined in the $(x, z)$ vertical plane, and three azimuthal anisotropy parameters: two deviation parameters $\epsilon_{d}, \eta_{d}$, and the parameter $\delta_{3}$ defined from the $x$-axis TI plane (Tsvankin, 1997). The horizontal velocity is related to the vertical P-wave velocity $v_{v}$ and to the NMO velocity $v_{n 1}$ in the $(x, z)$ plane through the equations given by

$$
\begin{aligned}
& v_{h 1}=v_{v} \sqrt{1+2 \epsilon_{1}}, \\
& v_{h 1}=v_{n 1} \sqrt{1+2 \eta_{1}} .
\end{aligned}
$$

The two deviation anisotropy parameters used in this parameterization are

$$
\begin{gathered}
\epsilon_{d}=\frac{1}{2}\left(\frac{v_{h 2}^{2}}{v_{h 1}^{2}}-1\right), \\
\eta_{d}=\frac{\epsilon_{d}-\delta_{d}}{1+2 \delta_{d}},
\end{gathered}
$$

where $v_{h 2}$ is the horizontal velocity in the $(y, z)$ vertical plane and $\delta_{d}$ is another deviation anisotropy parameter defined as 


$$
\delta_{d}=\frac{1}{2}\left(\frac{v_{n 2}^{2}}{v_{n 1}^{2}}-1\right),
$$

where $v_{n 2}$ is the NMO velocity in the $(y, z)$ vertical plane. The parameters $\epsilon_{d}$ and $\eta_{d}$, as well as $\delta_{3}$, measure the deviation from a VTI medium; therefore, if they are set to zero, the orthorhombic model reduces to VTI.

Figure 1 shows the radiation patterns of the parameterization described above, as a function of the scattering angle $\theta$, for different azimuths $\phi$. Here, $\theta$ is the angle between the source and receiver measured at the scattering point and $\phi$ is the source-to-receiver azimuth of the scattering point measured from the $(x, z)$ vertical plane. These radiation patterns correspond to perturbations from a horizontal reflector. They are also derived by assuming a small scatterer in a smoothly varying isotropic background medium (Masmoudi and Alkhalifah, 2016a). The effect of the background medium on the perturbation is expected to be small (He and Plessix, 2017). The resolution of the scattering point is governed by the diffraction tomography principles formula, which relates the model wavenumber $k_{m}$ to the frequency $\omega$ and the scattering angle $\theta$ (Devaney, 1984; Miller et al., 1987; Jin et al., 1992), and is given by

$$
\left|k_{m}\right|=2 \frac{\omega}{v_{0}} \cos \frac{\theta}{2}
$$

where $v_{0}$ is the velocity at the scattering point of the background isotropic model. From this relation, we can predict that data from large offsets (large scattering angles) should produce low-wavenumber updates, which are essential at the early stages of the model building process. On the other hand, small angles are associated with high-wavenumber updates. Keeping these facts in mind, we will infer some conclusions about the resolution of the parameters from the behavior of their radiation patterns.

As shown in Figure 1, the scattering potential of the horizontal velocity is independent from the scattering and azimuth angles. On the other hand, the scattering potential of $\epsilon_{1}$ and $\eta_{1}$ is stationary over azimuth angles. Moreover, $\epsilon_{1}$ is associated with small scattering angles, while, $\eta_{1}$ is associated with intermediate scattering angles. We may infer that velocity could be well-resolved, at least its low-wavenumber model information because of the trade-off with $\epsilon_{1}$ at small scattering angles. Because $\epsilon_{1}$ affects vertically traveling waves, we may only update its high-wavenumber information. This is assuming density and other amplitude effects are ignored. However, in real cases, there is a trade-off between $\epsilon_{1}$ and density. It has been suggested that $\epsilon_{1}$ could absorb density effects (e.g., Alkhalifah and Plessix, 2014; Guitton and Alkhalifah, 2016). Since $\eta_{1}$ has weak scattering potential, it could probably be fixed during the inversion. Moreover, the scattering potential of the azimuthal anisotropy parameters changes with scattering and azimuth angles: The main scattering of $\delta_{3}$ resides at $\phi=45^{\circ}$, whereas the other two parameters have their maximum scattering energy at $\phi=90^{\circ}$. Similarly to $\eta_{1}, \eta_{d}$ has weak scattering potential and might not be resolved. On the other hand, we have the chance to access the low-wavenumber a)
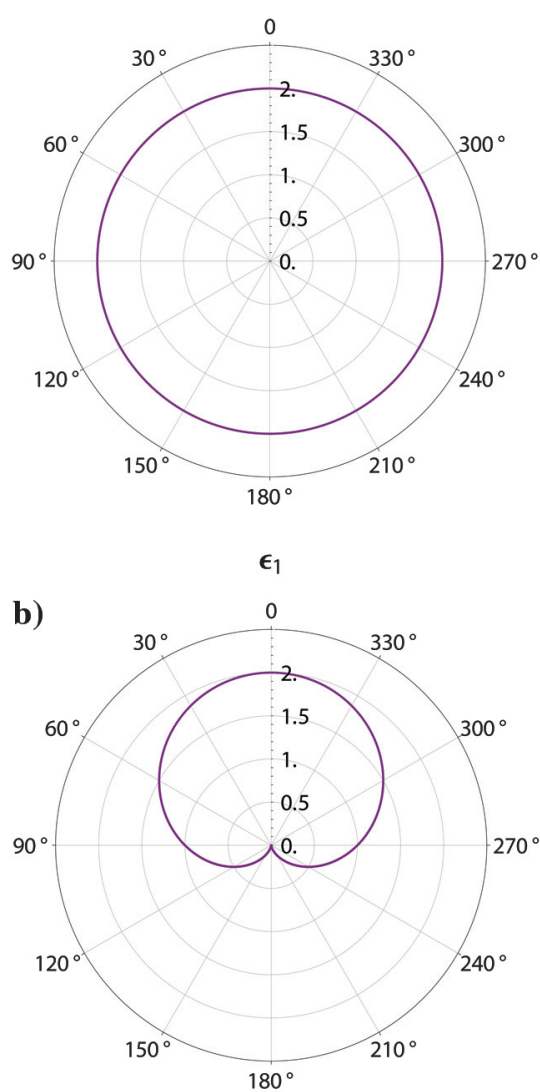

c)

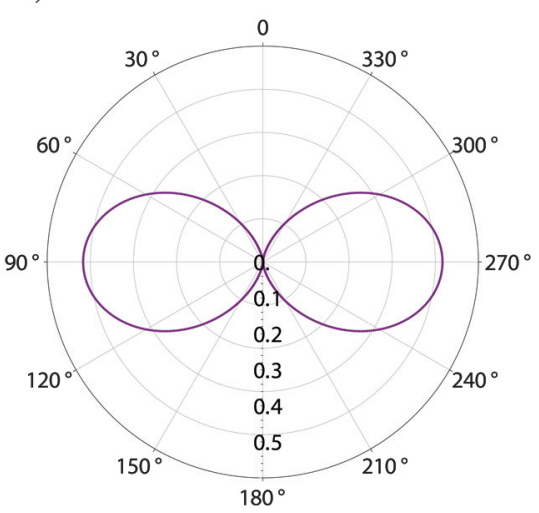

d)

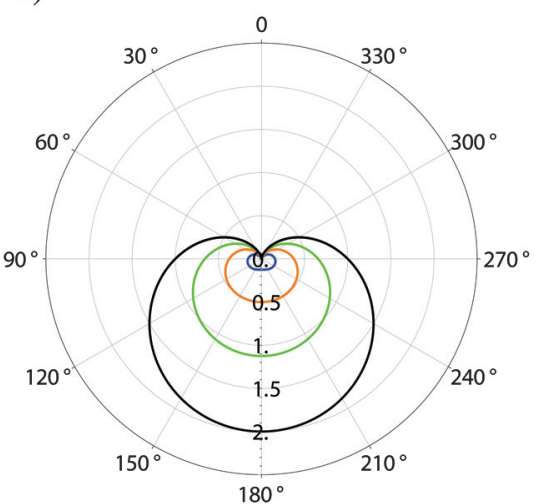

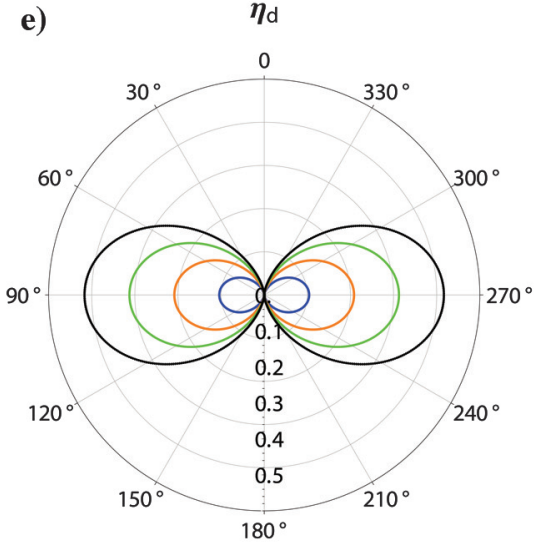

f)

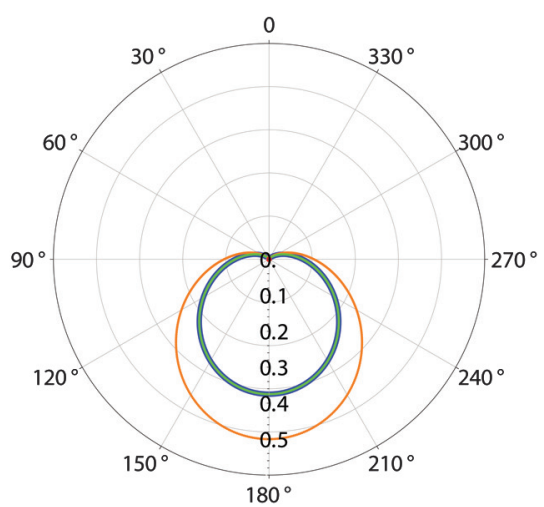

Figure 1. Radiation patterns for the parametrization proposed in Masmoudi and Alkhalifah (2016a) as a function of the scattering angle for different azimuth angles $\phi: 0^{\circ}-30^{\circ}-45^{\circ}-60^{\circ}-90^{\circ}-$ All azimuths. 
model update of $\epsilon_{d}$ and $\delta_{3}$ if we have large enough crossline offsets. However, we still have to mitigate the trade-offs between these two parameters and $v_{h 1}$.

\section{THEORY}

In FWI, we generally start with an initial model $m_{0}$ assumed to be close enough to the true model. The objective is to find a new model $m=m_{0}+\delta m$, which reduces the size of a misfit function in the framework of an optimization problem. The key ingredients in this process are an efficient modeling operator and a local differentiation approach, in which the gradient and potentially the Hessian operators are estimated. In this section, we first present our modeling approach and its adjoint formulation with the aim of deriving the gradients of the orthorhombic model parameters. Then, we describe a specific objective function we use in this study, which includes model regularization and structural constraints that we use in the real-data example.

\section{Forward modeling}

The acoustic wave equation for anisotropic media satisfies a linear pseudodifferential equation of the form

$$
\left[\omega^{2}-\Phi(\mathbf{x}, \mathbf{k})\right] u(\mathbf{k}, \omega)=0,
$$

where $u(\mathbf{k}, \omega)$ is the pressure wavefield, $\omega$ is the frequency, $\mathbf{k}$ is the wave vector, and $\Phi$ is the pseudodifferential phase operator. The phase operator can be obtained from the dispersion relation using the Christoffel equation (Alkhalifah, 2003). In the orthorhombic case, this yields the cubic polynomial (Song and Alkhalifah, 2013):

$$
-\Phi^{3}+a \Phi^{2}+b \Phi+c=0
$$

The polynomial coefficients $a, b$, and $c$ are given in Appendix A.

To solve equation 7 in the time-space domain, we propose similarly to Xu and Zhou (2014) and Xu et al. (2015) to decompose the pseudodifferential operator into an elliptic Laplacian operator $\nabla_{E}^{2}$ and a scalar operator $S(\mathbf{x})$ as follows:

$$
\partial_{t t} u(\mathbf{x}, t)-S(\mathbf{x}) \nabla_{E}^{2} u(\mathbf{x}, t)=0,
$$

where

$$
\left\{\begin{array}{c}
\nabla_{E}^{2} u(\mathbf{x}, t)=\partial_{x x} u+\left(1+2 \epsilon_{d}\right) \partial_{y y} u+\frac{1}{1+2 \epsilon_{1}} \partial_{z z} u \\
S(\mathbf{x})=\Phi(\mathbf{x}, \mathbf{n}) /\left(n_{x}^{2}+\left(1+2 \epsilon_{d}\right) n_{y}^{2}+\frac{1}{1+2 \epsilon_{1}} n_{z}^{2}\right)
\end{array}\right.
$$

where $\partial_{t t}$ is the second-order time derivative, $\partial_{x x}, \partial_{y y}$, and $\partial_{z z}$ are the second-order space derivatives, $n_{x}, n_{y}$, and $n_{z}$ are the components of the unit vector $\mathbf{n}$ of phase direction $\mathbf{n}=\mathbf{k} /|\mathbf{k}|$. In this elliptic decomposition, the ellipticity of the wavefield is handled by a slightly modified Laplacian operator, whereas the anellipticity is corrected via the scalar operator. Therefore, $S=1$ for an elliptic anisotropic media $\left(\eta_{1}=\eta_{d}=\delta_{3}=0\right)$. To compute the scalar operator in the space domain, we approximate the phase direction $\mathbf{n}$ from the components of $\nabla u /|\nabla u|$ (Xu and Zhou, 2014).

To test the accuracy of our modeling approach, we compare the wavefield solution from equation 9 with the low-rank approximation (Song and Alkhalifah, 2013), as well as with the traveltimes from the eikonal solution (Waheed et al., 2014). Figure $2 \mathrm{a}$ and $2 \mathrm{~b}$ shows wavefield snapshots in an orthorhombic medium with the homogeneous model parameters $v_{v}=2000 \mathrm{~m} / \mathrm{s}, \epsilon_{1}=0.2, \epsilon_{2}=0.4$, $\delta_{1}=0.1, \delta_{2}=0.15$, and $\delta_{3}=0.05$. Here, $\delta_{1}$ and $\delta_{2}$ are the Thomsen's (1986) parameters in the $(x, z)$ and $(y, z)$ vertical planes, respectively. These values correspond to $v_{h 1} \approx 2366, \epsilon_{1}=0.2$, $\epsilon_{d} \approx 0.143, \eta_{1} \approx 0.0833, \eta_{d} \approx 0.093$, and $\delta_{3}=0.05$ in the parameterization considered in this paper. The new wavefield solution matches the kinematics of the low-rank solution, as confirmed by comparison with the traveltimes from the eikonal solution. Obviously, our proposed modeling is free of S-wave artifacts. Figure 2c shows the corresponding $S$ operator. It is clear that $S$ corrects for the anellipticity of the wavefield because its maximum influence resides at $45^{\circ}$ propagation direction and is equal to one at the principal axes of symmetry.

\section{Adjoint formulation}

The adjoint state method provides an efficient way to derive the gradient of the FWI misfit function through the solution of an adjoint equation (Liu and Tromp, 2006; Plessix, 2006). For simplicity, we consider here the least-squares misfit function defined as a)

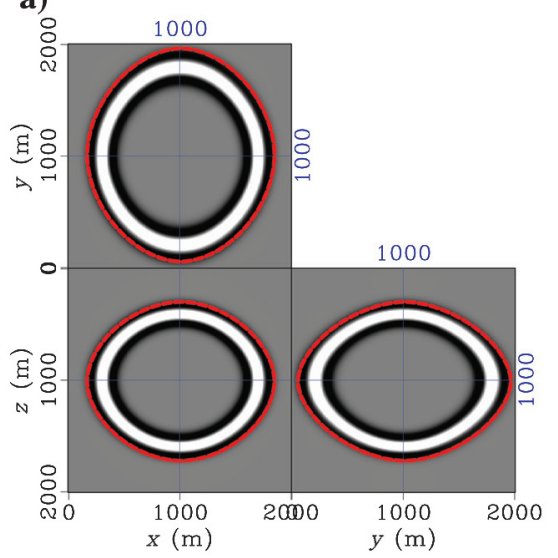

b)

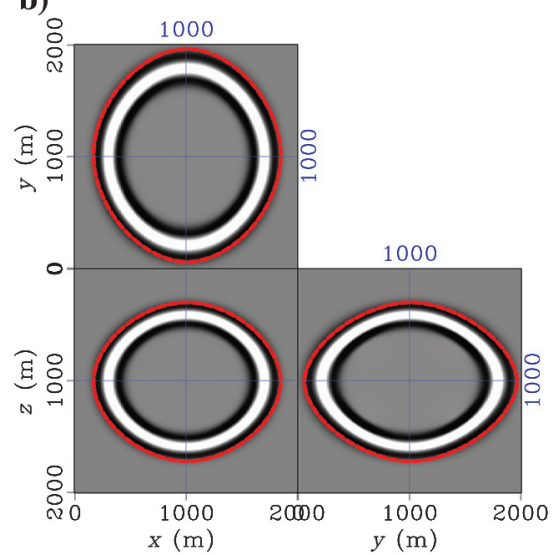

c)

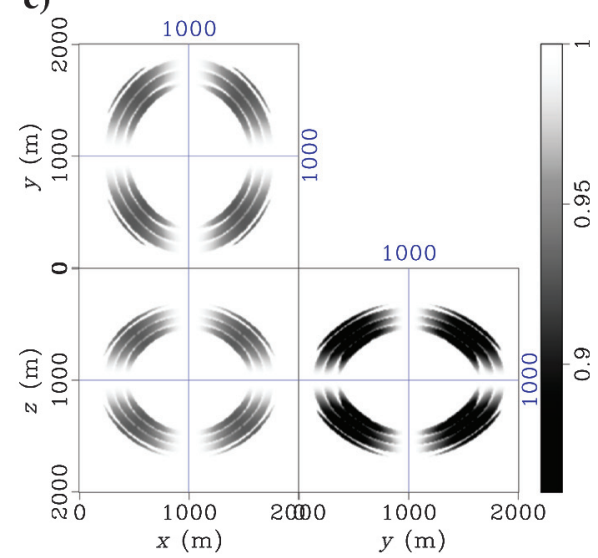

Figure 2. (a) Wavefield snapshots computed with the low-rank approximation, (b) the new modeling approach (equations 9 and 10), and (c) its corresponding S operator. The dashed red contours represent the eikonal solution. 


$$
J_{d}(m)=\frac{1}{2} \sum_{r} \int_{0}^{T}\left\|u\left(\mathbf{x}_{r}, t\right)-d\left(\mathbf{x}_{r}, t\right)\right\|^{2} d t
$$

where $m$ denotes the model parameters, $\left[\begin{array}{ll}0 & T\end{array}\right]$ denotes the time series of interest, $\mathbf{x}_{r}$ are the receiver locations, $d\left(\mathbf{x}_{r}, t\right)$ is the observed data, and $u\left(\mathbf{x}_{r}, t\right)$ is the modeled data satisfying the wave equation 9 , rewritten with a source term $f$ :

$$
\partial_{t t} u(\mathbf{x}, t)-S(\mathbf{x}) \nabla_{E}^{2} u(\mathbf{x}, t)=f,
$$

with initial conditions given by
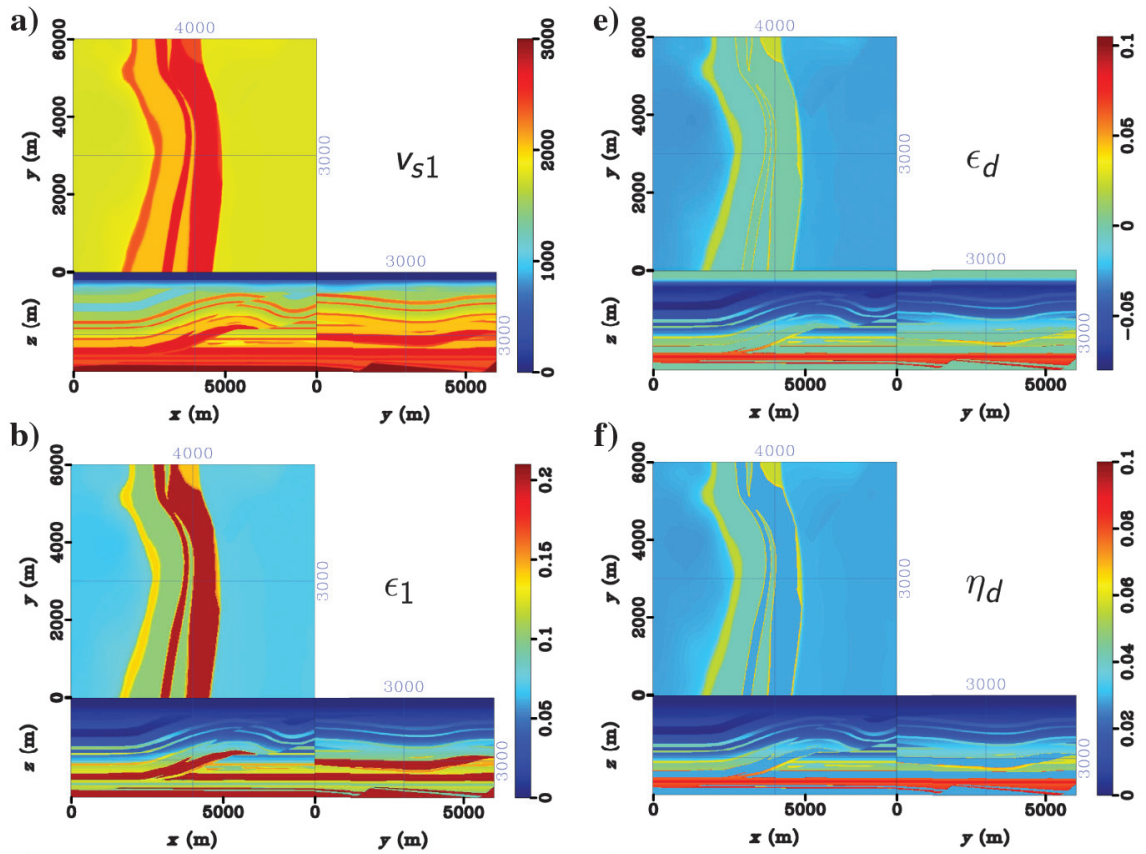

$$
u(\mathbf{x}, 0)=0, \quad \partial_{t} u(\mathbf{x}, 0)=0 .
$$

Using the adjoint state method, we obtain the adjoint wavefield and the gradients of the misfit function 11 . In this case, the adjoint wavefield $\lambda$ satisfies the following equation:

$$
\begin{aligned}
& \left(1 / v_{h 1}^{2}\right) \partial_{t t} \lambda(\mathbf{x}, t)-S(\mathbf{x}) \nabla_{E}^{2} \lambda(\mathbf{x}, t) \\
& \quad-\nabla_{E}^{2} u(\mathbf{x}, t) \nabla \lambda(\mathbf{x}, t)(\partial S / \partial \nabla u)^{T} \\
& \quad=\sum_{r}\left[u\left(\mathbf{x}_{\mathbf{r}}, T-t\right)-d\left(\mathbf{x}_{\mathbf{r}}, T-t\right)\right] \delta\left(\mathbf{x}-\mathbf{x}_{\mathbf{r}}\right),
\end{aligned}
$$
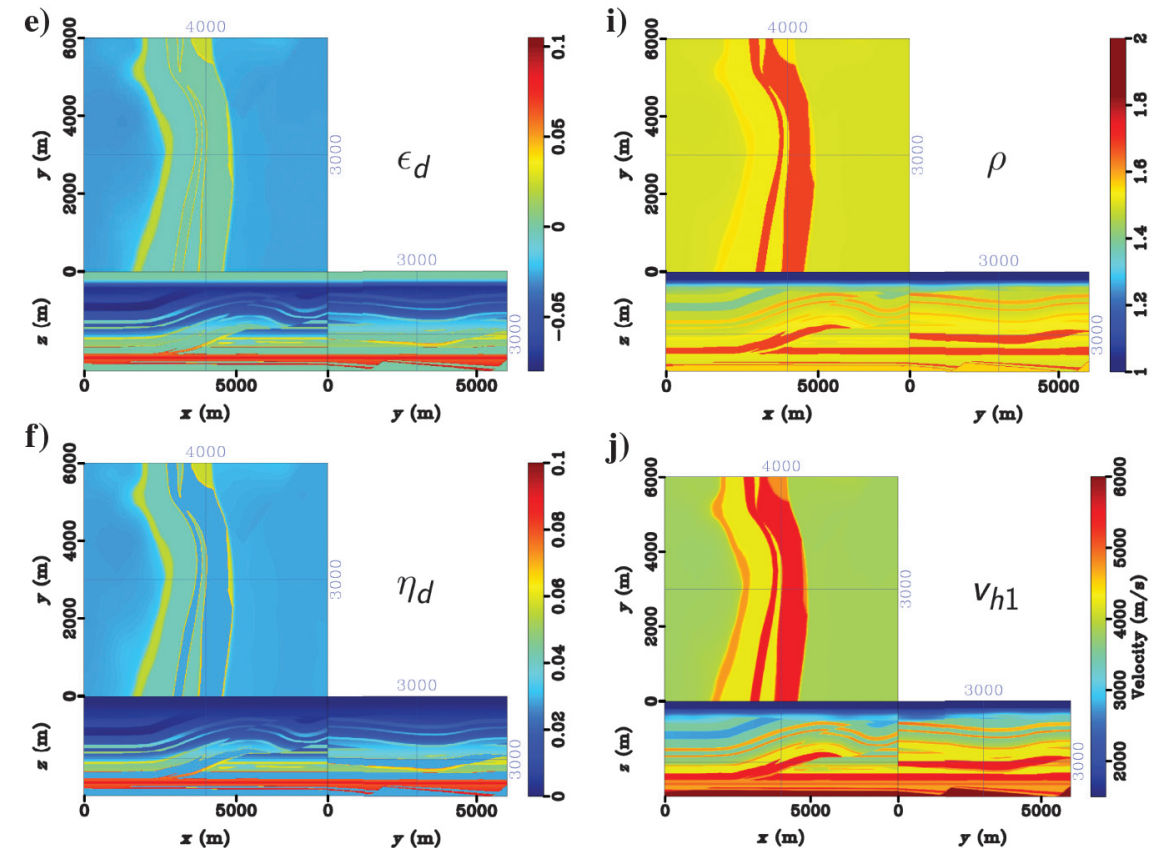
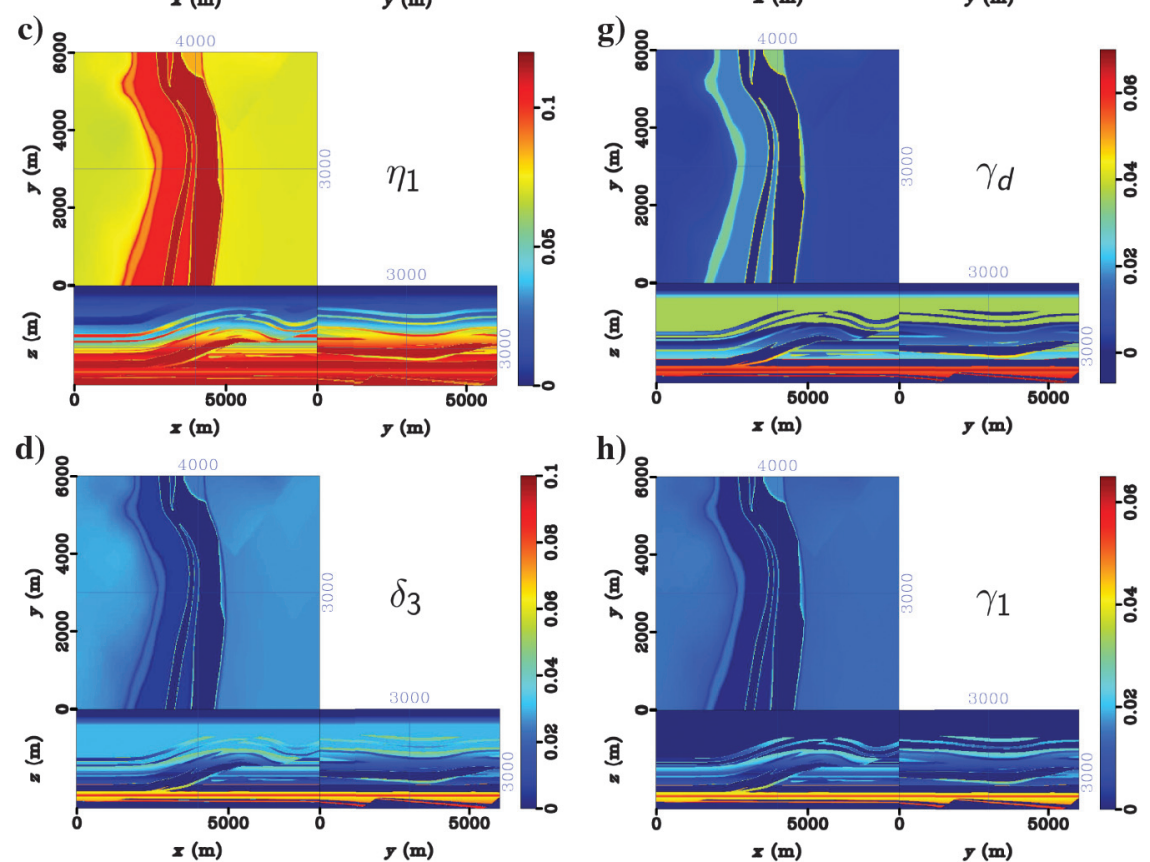

Figure 3. An elastic variable density orthorhombic model parameters obtained from the SEG-EAGE overthrust model. 
with initial conditions given by

$$
\lambda(\mathbf{x}, T)=0, \quad \partial_{t} \lambda(\mathbf{x}, T)=0,
$$

where $\delta\left(\mathbf{x}-\mathbf{x}_{\mathbf{r}}\right)$ is the Dirac delta function. The adjoint source in equation 14 is the data residual obtained by subtracting the observed and modeled data set at the receiver locations. The gradients with Appendix B. The partial derivatives of $S$ in equation 14 and in the gradients (equations B-1-B-6) are not easy to obtain from the solution of the cubic polynomial 8 . Therefore, we approximate its P-wave solution using a first-order Taylor expansion. Specifically, by assuming the anisotropy parameters to be small, we expand the solution of $\Phi$ in terms of $\epsilon_{1}, \epsilon_{d}, \eta_{1}, \eta_{d}$, and $\delta_{3}$ as follows:

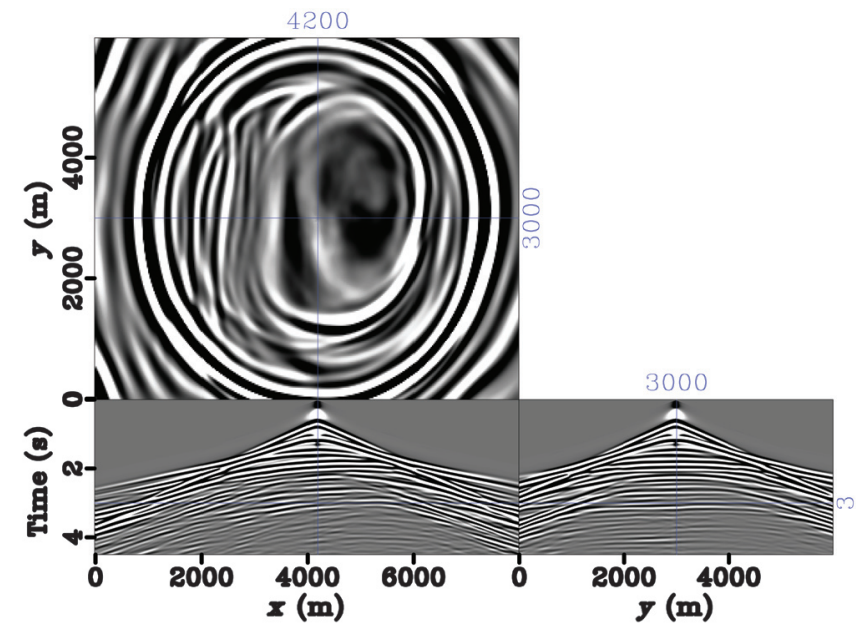

Figure 4. Example of one shot gather generated from the overthrust model shown in Figure 3. respect to the parameterization considered in this paper are given in

$$
\Phi \approx \Phi_{0}+\Phi_{\epsilon_{d}} \epsilon_{d}+\Phi_{\epsilon_{1}} \epsilon_{1}+\Phi_{\eta_{1}} \eta_{1}+\Phi_{\eta_{d}} \eta_{d}+\Phi_{\delta_{3}} \delta_{3},
$$

where $\Phi_{0}, \Phi_{\epsilon_{1}}, \Phi_{\epsilon_{d}}, \Phi_{\eta_{1}}, \Phi_{\eta_{d}}$, and $\Phi_{\delta_{3}}$ are the coefficients in this expansion. By inserting equation 16 into the cubic polynomial 8 , one can solve for the coefficients and obtain an approximation of $\Phi$, given by

$$
\begin{aligned}
\Phi & \approx 1+2 k_{y}^{2}\left(k_{y}^{2}+k_{z}^{2}\right) \epsilon_{d}-2 k_{z}^{2} \epsilon_{1}-2 k_{z}^{2}\left(k_{x}^{2}+k_{y}^{2}\right) \eta_{1} \\
& +2 k_{y}^{2} k_{z}^{2} \eta_{d}+2 k_{x}^{2} k_{y}^{2} \delta_{3},
\end{aligned}
$$

where $k_{x}, k_{y}$, and $k_{z}$ are the components of the wave vector $\mathbf{k}$. Using the new approximation 17, we obtain expressions for the partial derivatives of $S$ with respect to the model parameters (see Appendix B).

\section{The objective function}

In practice, FWI may require some kind of regularization and constraints to reduce the model null-space and to steer the inversion toward geologically meaningful models. In the scope of this paper, we rely on an objective function containing a data-misfit term, a damped TV term, and a structural constraint term:

$$
J(m)=J_{d}(m)+\alpha J_{\mathrm{TV}}(m)+\beta J_{\mathrm{cons}}(m),
$$

where $\alpha$ and $\beta$ control the contribution of the penalty terms. To relax the requirement for amplitude matching, we use the normalized crosscorrelation objective function instead of the least-square misfit function. The normalized crosscorrelation evaluates the similarity between the observed and modeled data (Choi and Alkhalifah, 2012):

$$
J_{d}(m)=-\hat{u} \cdot \hat{d}
$$
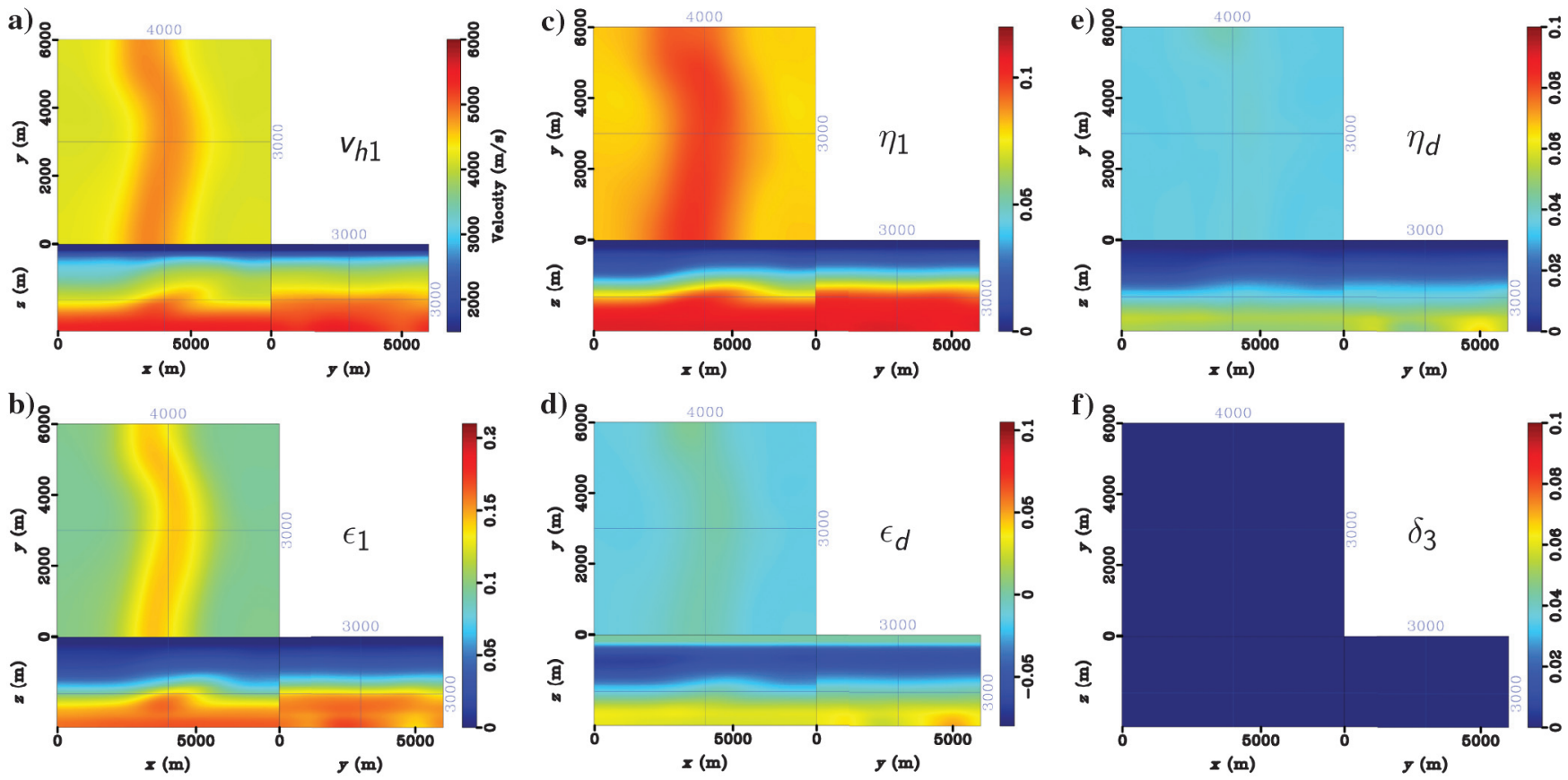

Figure 5. Initial model parameters used in the overthrust model inversion. 
where $\hat{d}=d /\|d\|$ is the normalized observed data and $\hat{u}=u /\|u\|$ is the normalized modeled data. Therefore, we replace the adjoint source in the adjoint wave equation 14 by the following adjoint source (Choi and Alkhalifah, 2012):

$$
1 /\|u\|((\hat{d} \cdot \hat{u}) \hat{u}-\hat{d})
$$

Moreover, we use a smooth TV as a penalty in the objective function (Guitton, 2012):

$$
J_{\mathrm{TV}}(m)=\int \sqrt{\|\nabla m\|^{2}+e^{2}} d x,
$$

where $e$ mitigates the singularity in the gradient. Finally, we use a cross-gradient constraint (Gallardo and Meju, 2003; Hu et al., 2009) to enforce the structural similarity between any two model parameter combinations $m_{i}$ and $m_{j}$ :

$$
J_{\text {cons }}(m)=\int\left|\nabla m_{i} \times \nabla m_{j}\right| d x .
$$
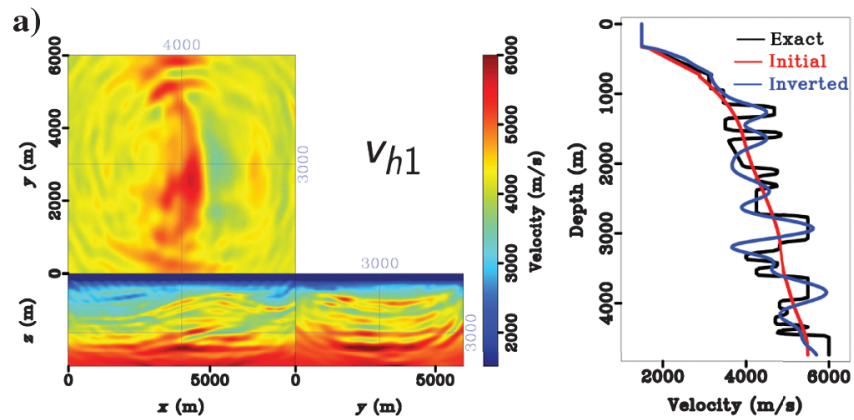

b)
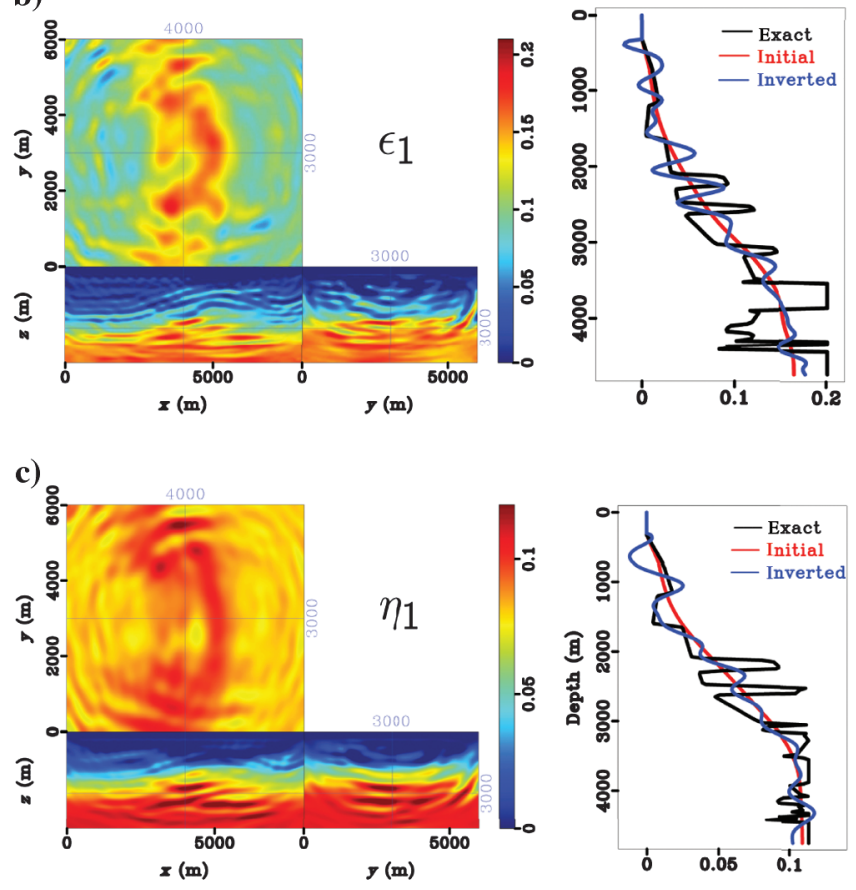

In the real-data application, as we will explain later, the structural similarity constraint is applied to $\epsilon_{1}$ and $\epsilon_{d}$.

\section{IMPLEMENTATION}

We developed a 3D finite-difference modeling algorithm and the associated FWI infrastructure valid for an acoustic constant density orthorhombic medium. Our forward modeling relies on solving equation 9 using an explicit time-domain finite-difference algorithm. The main extra step in our modeling approach compared with an isotropic wavefield propagator is the computation of the scalar operator $S(\mathbf{x})$. Furthermore, we use a second-order finite-difference scheme for time stepping and a fourth-order scheme for the space derivatives. The boundary condition for $3 \mathrm{D}$ modeling requires computationally efficient implemetation, with as little extension to the computational domain as possible. In this study, we rely on the absorbing boundary conditions (Clayton and Engquist, 1977), which compute simplified one-way wave equations to predict the wavefield beyond the boundary. To compute the gradients, we use a strategy based on making the forward (source) wavefield available at the same time step as the adjoint wavefield. We implement here the boundary saving approach (Dussaud et al., 2008; Yang et al.,
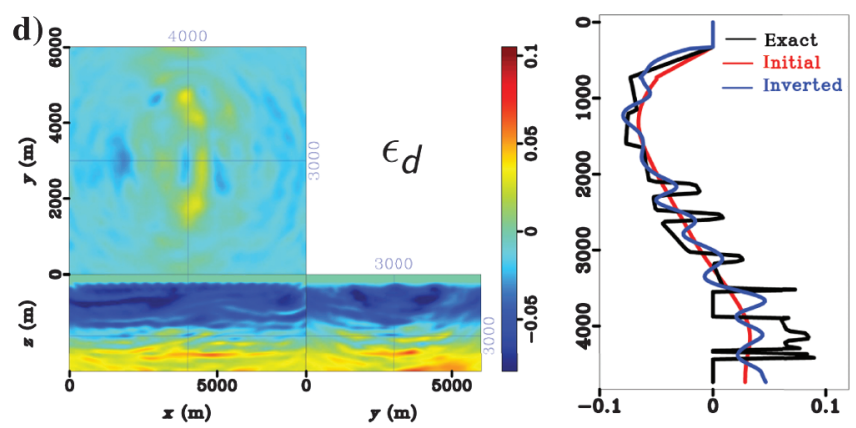

e)
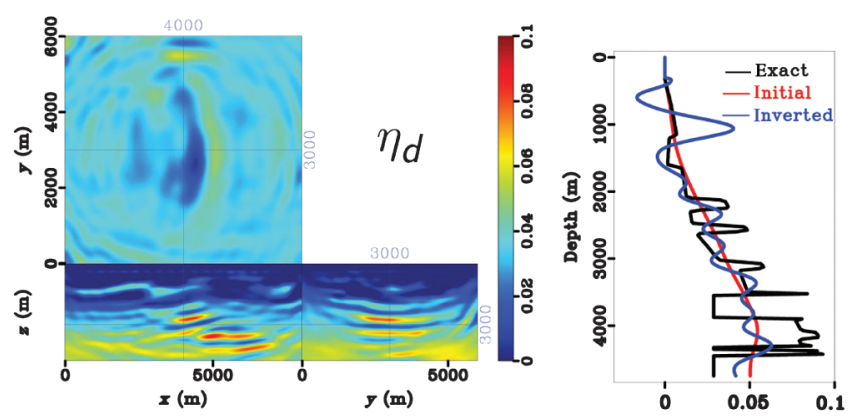

f)
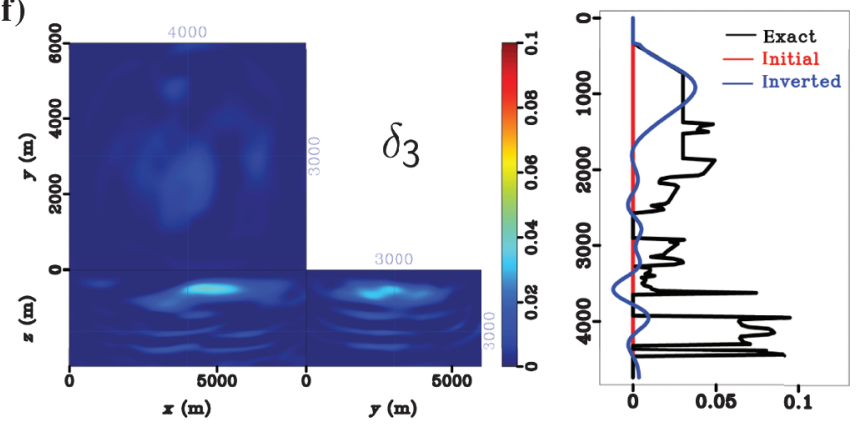

Figure 6. Inverted model parameters and their vertical profiles at ( $x=4 \mathrm{~km}, y=3 \mathrm{~km})$. 
2014), in which the final two time steps of the forward wavefield as well as data recorded during the forward propagation step at the boundary of the computation domain are used to run the source wavefield backward in time. During the backward propagation, we use the regenerated source wavefield information to compute the diagonal of the approximate Hessian (Shin et al., 2001). The diagonal of the approximate Hessian (the pseudo-Hessian) is then used to precondition the gradients. Such preconditioning approximately compensates for illumination effects. In other words, it enhances the gradient where the amplitude of the source wavefield is weak and reduces it where the wavefield amplitude is large. Moreover, we improve the descent direction by using the nonlinear conjugate gradient algorithm. Finally, global step lengths that scale each parameter gradient can be estimated using a line-search method in which the second-order approximation of the objective function is evaluated (Pica et al., 1990; Sambridge et al., 1991). This estimation step requires solving an additional forward modeling problem for each parameter, as well as solving a linear system of equations (Masmoudi and Alkhalifah, 2017). Although this estimation can slightly mitigate some of the trade-off issues, in practice, we find it challenging to apply for our objective function (equation 18). Therefore, in this study, we rely on simple decreasing step lengths as a function of iterations.

Our FWI code is designed to run efficiently on large highperformance computing systems by using the Message Passing
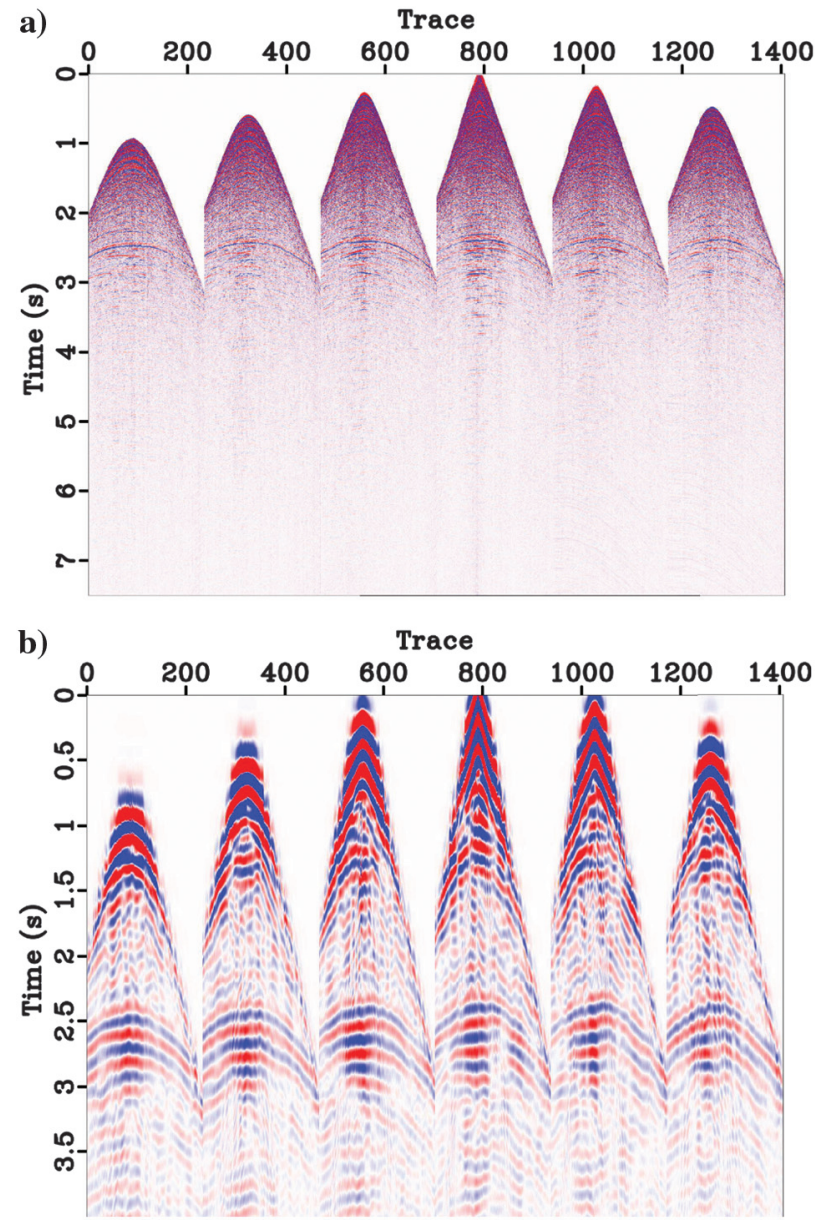

Figure 7. (a) Example of shot gathers from six different cables and (b) the same shot gathers after a band-pass filter and time windowing.
Interface library across the shots and OpenMP to accelerate the computation within a compute node across the available cores of the processor. Typically, one shot is run on one compute node. When few nodes are available, we may run in parallel several shots on each node. When this is not possible due to limited memory or a small number of cores per node, we may sequentially run several shots on the same node. Proper synchronization between the compute nodes and input/output management are essential to maintain the code scalability. Retaining as much as possible data in local memory, rather than writing and reading from the disk, preserves the performance of the FWI code. In our implementation, a compute node holds in local memory the model parameters and the observed data for one shot and then it computes the forward wavefield and the adjoint source; it also stores the recorded source wavefield at the boundary of the computation domain and then it computes the adjoint wavefield and crosscorrelates it with the regenerated source wavefield to form the gradients and the pseudo-Hessians. Once all local gradients and pseudo-Hessians are computed, they are spread over all compute nodes and stacked to form a global gradient and a global pseudo-Hessian. Therefore, to reduce the communication through the interconnect and to maintain the scalability of the code, the main information that needs to travel across the network is the gradient and the pseudo-Hessian. Finally, one single node is writing to the disk the updated model at every iteration.

\section{NUMERICAL EXAMPLES}

\section{An SEG-EAGE overthrust model inversion}

We first show an application of the acoustic orthorhombic inversion on a modified 3D SEG-EAGE overthrust model. By scaling the original P-wave velocity, we generate the elastic variable density orthorhombic model given in Figure 3. The parameters in Figure 3 correspond to the elastic parameterization proposed in $\mathrm{Oh}$ and Alkhalifah (2016b). In addition, we add a water layer of $375 \mathrm{~m}$ and a transition bumping layer of $375 \mathrm{~m}$ on top of the original model. To reduce the computational time, we decrease the total size of the original model and end up using an inversion domain of $8 \times 6 \mathrm{~km}$ laterally and $4.75 \mathrm{~km}$ in depth. We use this elastic orthorhombic model to generate streamer data. Specifically, 60 shots are distributed on the water surface (at $25 \mathrm{~m}$ depth) in three crossline ( $y$ axis) directions at $1.5,3$, and $4.5 \mathrm{~km}$, with 20 shots in each line spaced by $400 \mathrm{~m}$. Data are collected from receivers on the water surface placed at a $25 \mathrm{~m}$ depth at all the grid points of the computational domain. One shot gather example is shown in Figure 4. A $12.5 \mathrm{~m}$ grid spacing in all three dimensions is used to generate the observed data; however, a $25 \mathrm{~m}$ grid spacing is used in our acoustic inversion. The initial model is obtained from smoothing the true acoustic model parameters except $\delta_{3}$, which is set to zero (see Figure 5). In practice, MVA or tomography combined with log information are necessary to obtain such an initial model; $\delta_{3}$ is generally hard to estimate even with these conventional techniques.

In terms of inversion strategy, we rely on the normalized crosscorrelation objective function (see equation 19). We also use a hierarchical selection of frequencies by successively inverting three frequency bands $(2-6,2-8$, and $2-12 \mathrm{~Hz})$. Moreover, we use a multistage model inversion strategy: We update first the isotropic parameter $v_{h 1}$ (20 iterations) at the first frequency band, then the VTI model by including $\eta_{1}$ and $\epsilon_{1}$ (five iterations) on the second frequency band, and finally the orthorhombic model by including $\epsilon_{d}, \eta_{d}$, 
and $\delta_{3}$ (five iterations) on the last frequency band. One can also simultaneously update all parameters in one stage. This strategy requires proper gradient preconditioning using the second-order derivatives of the objective function, specifically the Hessian matrix (Pratt et al., 1998; Pan et al., 2016). However, the size of the Hessian matrix makes this computationally challenging (Métivier et al., 2014). On the other hand, if we update parameters that have strong effect on the data first then include additional parameters with less effect at later stages, we will have the potential to reduce the crosstalk between the parameters. In the selected parameterization, we have such an opportunity because there is a continuity in the scattering potential of the model parameters as we move from higher symmetry anisotropy to lower ones (Masmoudi and Alkhalifah, 2016b).

Figure 6 shows the inverted model as well as vertical profiles at the center of the model comparing initial, true, and inverted model parameters. Figure 6 a shows that the horizontal velocity is generally well recovered. Indeed, most of the overthrust model structures are captured at their correct position. The parameter $\epsilon_{1}$, which affects the data mainly at narrow scattering angles, is not well-recovered here (Figure 6b). Some of the true $\epsilon_{1}$ structures have been captured; however, additional events leaked to this parameter. Because $\epsilon_{1}$ and density have a similar influence on the data, it is possible that $\epsilon_{1}$ has absorbed the density effect. On the other hand, the shallow part of $\epsilon_{d}$ (up to $3 \mathrm{~km}$ depth) is relatively well-recovered as shown in Figure 6d. This is in agreement with the radiation pattern analysis, which predicts that large offsets are necessary to update this parameter. Similar conclusions hold for the inverted $\delta_{3}$. The main shallow variations (up to $1.5 \mathrm{~km}$ depth) at the center of the model are retrieved (Figure 6f). Finally, $\eta_{1}$ and $\eta_{d}$ are not well-recovered (Figure $6 \mathrm{c}$ and $6 \mathrm{e}$ ), as predicted, by the radiation patterns analysis and some previous studies pin-pointing the weak influence of $\eta$ on surface seismic data as a scatterer in this parameterization (Alkhalifah, 2016; Alkhalifah and Guitton, 2016).

We further explain the low-resolution of the inverted $\eta_{1}$ and $\eta_{d}$ by the potential trade-offs with the elastic parameters. Indeed, Oh and Alkhalifah (2016b) and Kazei and Alkhalifah (2018) analyze the scattering radiation patterns of the nine orthorhombic elastic parameters; six of them are the acoustic parameters that we are interested in here, and the three others are $v_{s 1}, \gamma_{1}$, and $\gamma_{d}$. Their studies reveal strong trade-offs between $v_{s 1}$ and $\eta_{1}$, as well as between $\eta_{d}$ and $\gamma_{d}$ when using PP and PS surface seismic data. Therefore, in our acoustic model inversion using elastic data, $\eta_{1}$ and $\eta_{d}$ might have absorbed some elastic effects due to these two additional parameters.

\section{Application to real data from the North Sea}

We test the 3D acoustic orthorhombic FWI on the Volve data from the North Sea. These data are acquired on the seafloor (at a $90 \mathrm{~m}$ depth) using 12 cables, with $400 \mathrm{~m}$ separation. Each cable contains 240 receivers, separated by $25 \mathrm{~m}$. The source vessel has provided shots over an area extending $3 \mathrm{~km}$ to the left and right of each cable. The maximum offset in the inline direction is $5 \mathrm{~km}$, whereas the maximum offset in the crossline direction is $1.2 \mathrm{~km}$. The short offset, mainly in the crossline direction, will limit our ability to invert for some of the longwavelength information of parameters describing azimuthal information. Moreover, the data have been processed by the data owners for migration purposes. An example of a vertical-component shot gather is shown in Figure 7a. One can notice that parts of the diving waves are removed. On the other hand, a tomographic VTI model (see Figure 8) was provided.

We invert for the vertical-component data with frequencies ranging between 2 and $10 \mathrm{~Hz}$. Figure $7 \mathrm{~b}$ shows one shot gather example used in this inversion after a band-pass filter and a time windowing
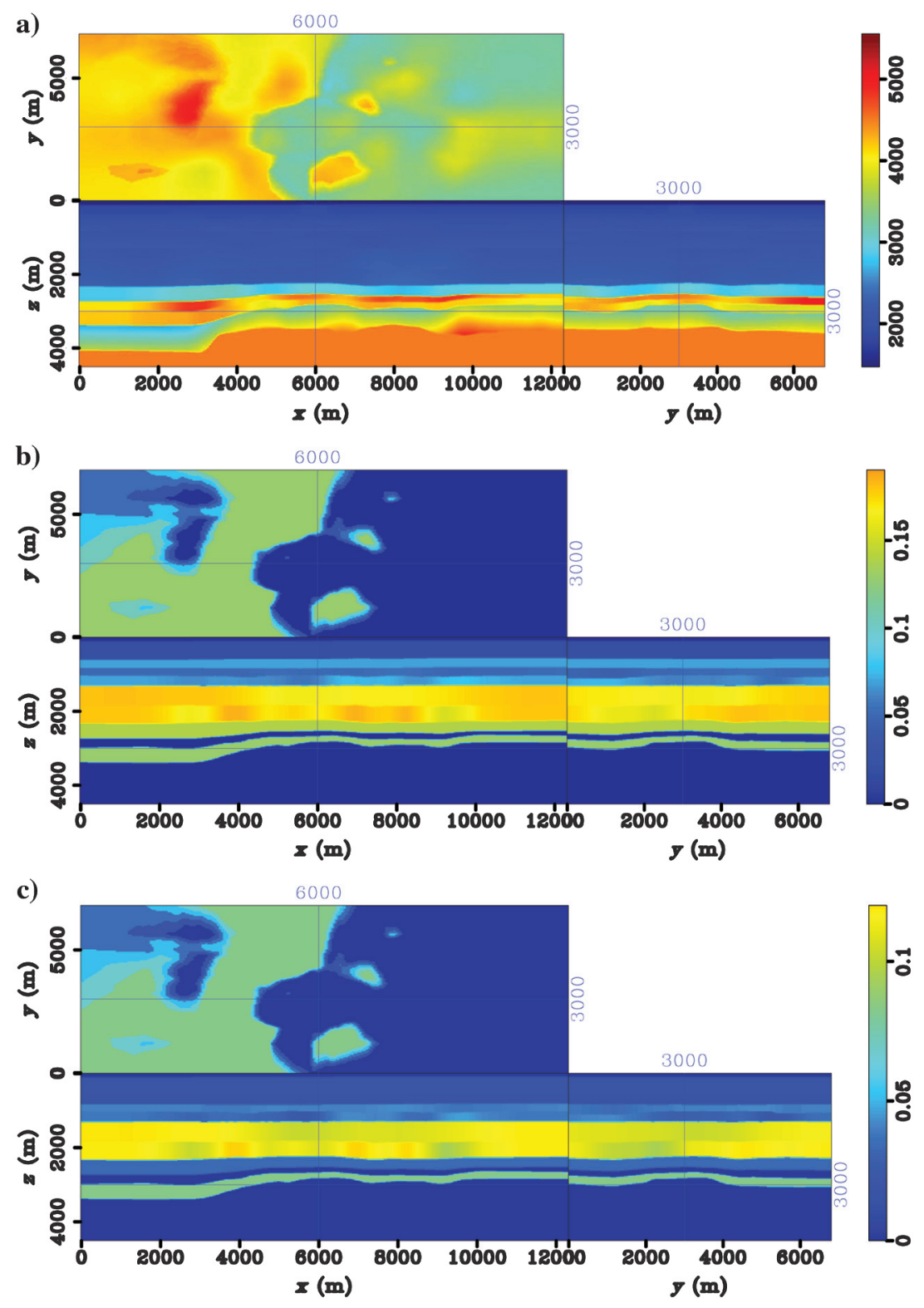

Figure 8. The VTI tomographic model parameters: (a) $v_{h 1}$, (b) $\epsilon_{1}$, and (c) $\eta_{1}$. 
up to 4 seconds of the original data. We smooth the tomographic model, as shown in Figure 9, and use it as an initial model for FWI. The size of the model is 12.3 (inline), 6.8, (crossline) and $4.5 \mathrm{~km}$ (depth). The initial velocity corresponds to a generally increasing velocity with depth with a mild low-velocity zone at approximately $3 \mathrm{~km}$ in the target reservoir area (Szydlik et al., 2007). Figure $9 \mathrm{~b}$ and 9c shows the initial $\epsilon_{1}$ and $\eta_{1}$ parameters. These two parameters reveal significant anisotropy, especially approximately $2 \mathrm{~km}$ depth. We set $\epsilon_{d}, \eta_{d}$, and $\delta_{3}$ to zero as azimuthal anisotropy information is not available. Given the short crossline offset, the influence of $\eta_{d}$ and $\delta_{3}$ on the data is expected to be weak; therefore, we do not update these two parameters and we focus only on the inversion of the three VTI parameters $\left(v_{h 1}, \epsilon_{1}\right.$, and $\left.\eta_{1}\right)$ and the azimuthal anisotropy parameter $\epsilon_{d}$.

At every FWI iteration, we randomly select 200 shots from 17123 available shots so as to limit the source and receiver imprints on the inverted model and to reduce the computational cost (Diaz and Guitton, 2011). We invert velocity first (20 iterations), and then we simultaneously update all parameters in a second stage (10 iterations). To resolve sharp interfaces, we apply TV regularization to
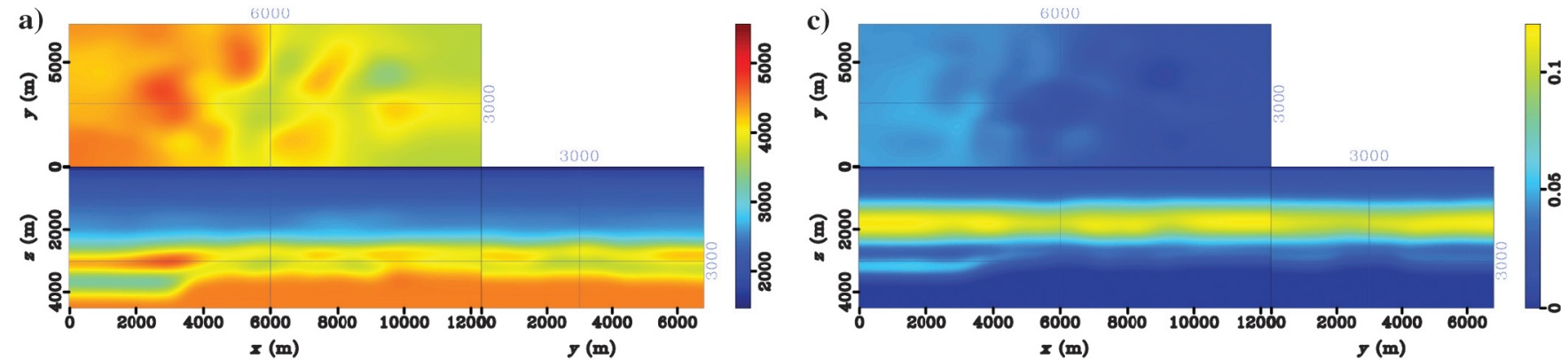

b)

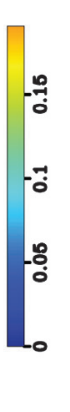

d)
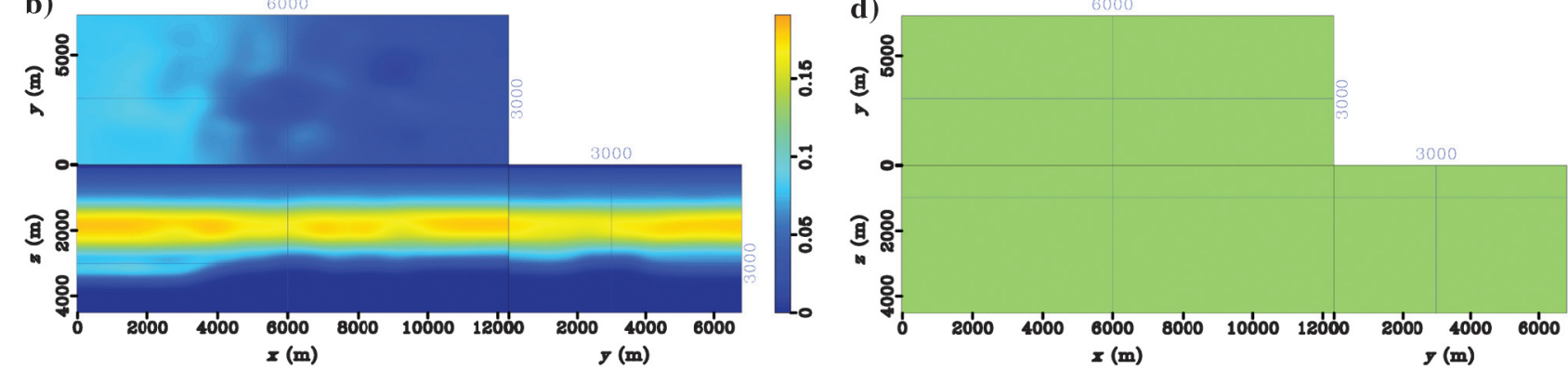

Figure 9. The initial model parameters: (a) $v_{h 1}$, (b) $\epsilon_{1}$, (c) $\eta_{1}$, and (d) $\epsilon_{d}$.

a)

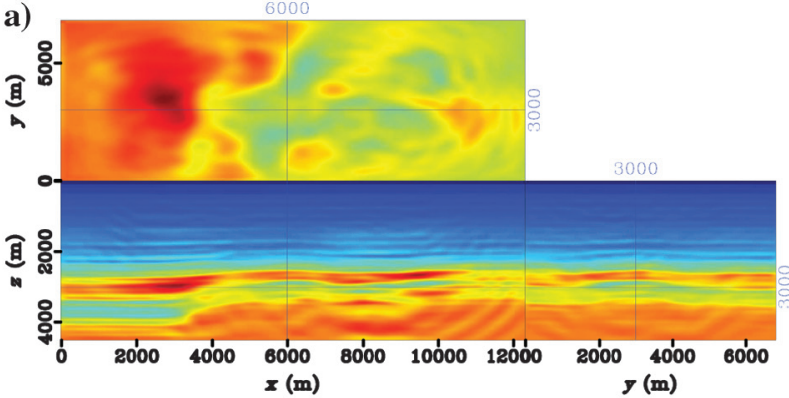

b)

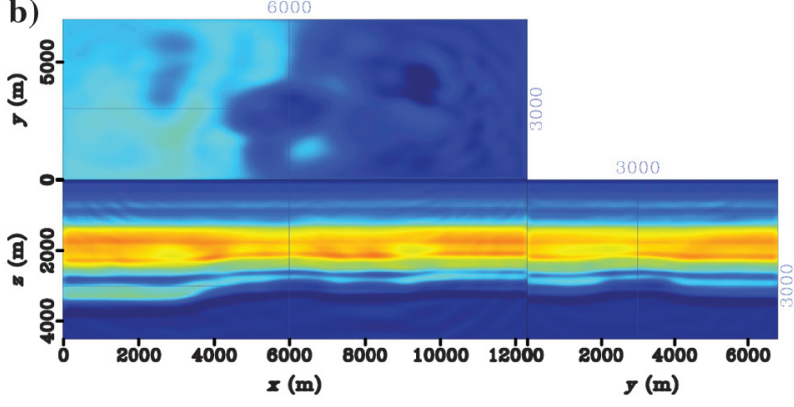

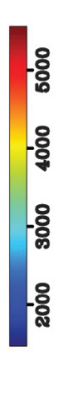

c)

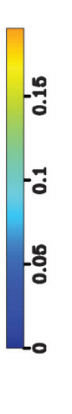

d)
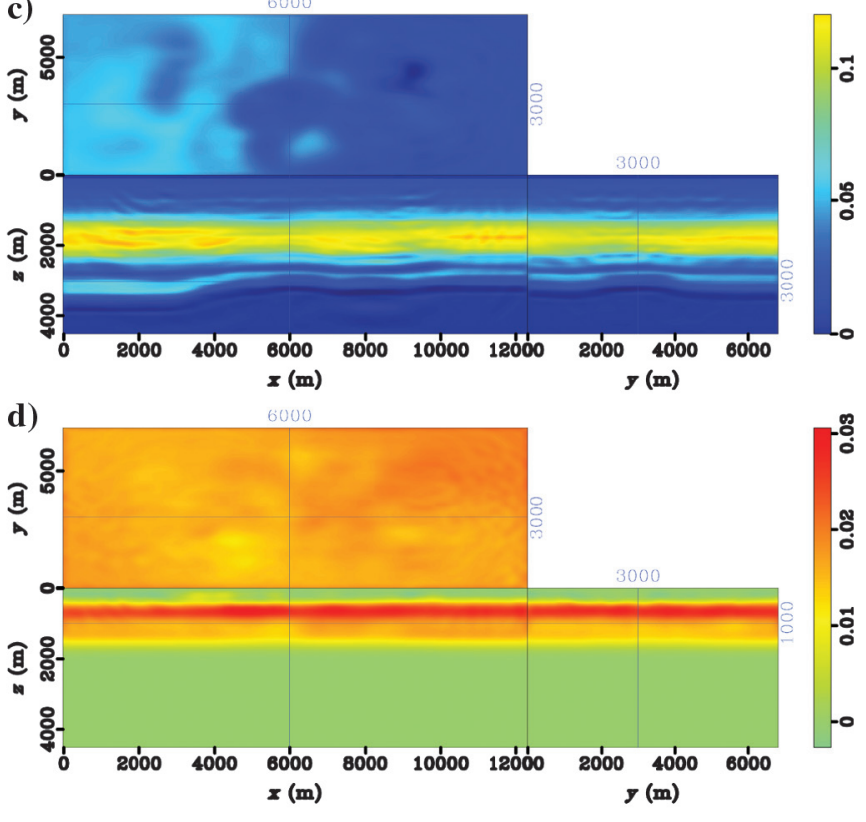

Figure 10. The inverted model parameters: (a) $v_{h 1}$, (b) $\epsilon_{1}$, (c) $\eta_{1}$, and (d) $\epsilon_{d}$. 
the inversion (see equation 21). Moreover, we apply structural constraints (see equation 22) to $\epsilon_{d}$ by constraining its shallow structural layers to be similar to $\epsilon_{1}$. The consistency of the structural layering between anisotropy parameters is generally expected. In addition, the deeper part of $\epsilon_{d}$ (less than $2 \mathrm{~km}$ depth) is not updated.

The inverted parameters are shown in Figure 10. From the inverted velocity (Figure 10a), one can clearly notice the low velocity (at $3 \mathrm{~km}$ depth), which corresponds to the target reservoir area. In

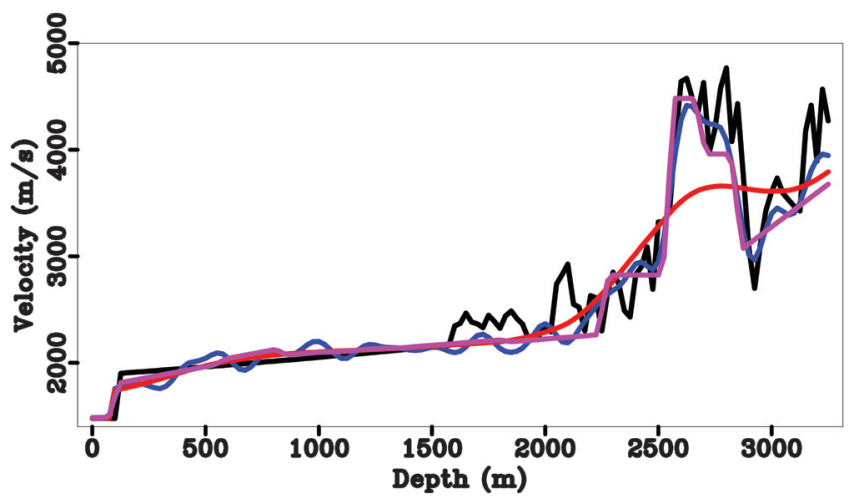

Figure 11. A vertical profile of the initial (red), tomographic (rose), inverted (blue), and the well-log (black) velocity extracted from the well location.
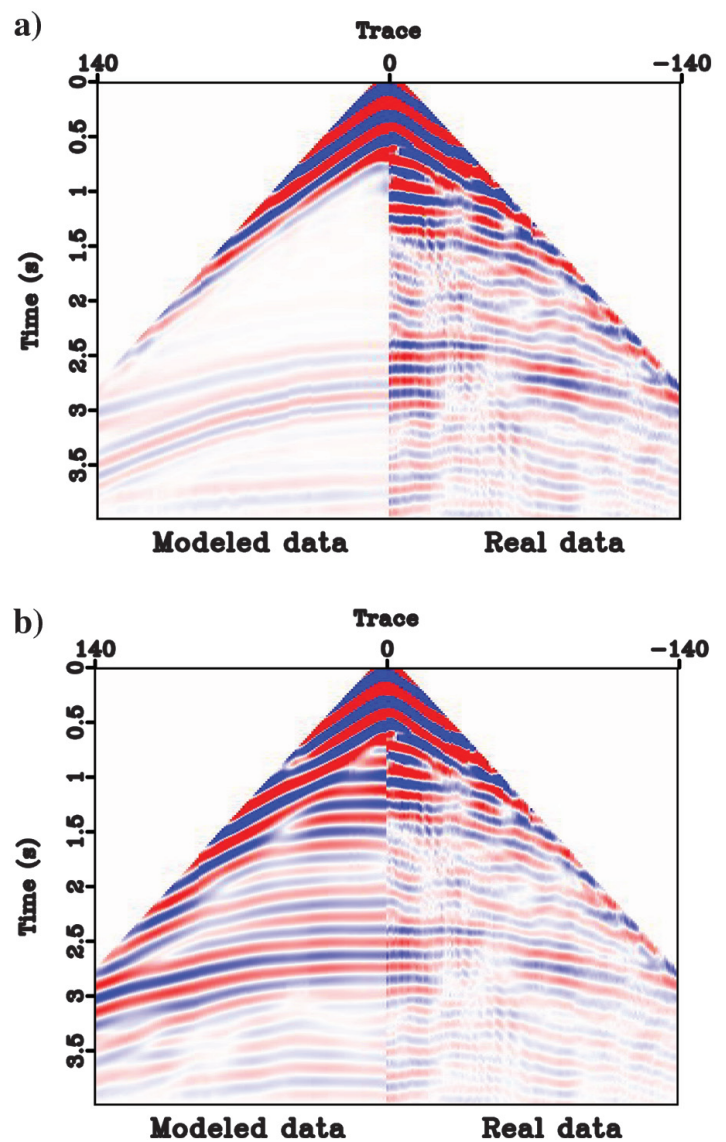

Figure 12. A shot gather comparing (a) the initial modeled data and (b) the final modeled data with the real data. the inverted $\epsilon_{1}$ and $\eta_{1}$, a thin and low anisotropy layer (almost an isotropic layer) at approximately $3 \mathrm{~km}$ depth is recovered (see Figure 10b and 10c). This layer is consistent with the lowvelocity layer in the reservoir area. Moreover, higher resolution details and sharper boundaries are obtained in the inverted three VTI parameters. On the other hand, we favor smooth updates for the azimuthal anisotropy parameter $\epsilon_{d}$ (Figure 10d) as predicted by the analytical analysis and the synthetic data inversion. A slightly deviating well of the vertical P-wave velocity (Figure 11) allows us to validate the overall inversion results. The well is located at approximately $8.2 \mathrm{~km}$ in the inline and $3 \mathrm{~km}$ in the crossline directions. Also included in Figure 11 are the initial, tomographic, and inverted vertical velocity. The inverted velocity is consistent with the well-log information and provides a better fit than the tomographic result, especially at depth. However, the high-resolution variations at approximately $2 \mathrm{~km}$ depth are not captured in our inverted model. Those details may require higher frequencies to be captured. Furthermore, we show in Figure 12 an initial and a final modeled shot gather using the starting and final models. Data generated with the inverted model contain many reflections that match the real shot gather. Finally, the common image angle gathers (CIGs) from different locations along an inline direction (at $y=3 \mathrm{~km}$ ) and a crossline direction (at $x=6 \mathrm{~km}$ ) are shown in Figures 13 and 14 . We notice that the inverted model better flattens the CIGs compared with the initial model at several locations.
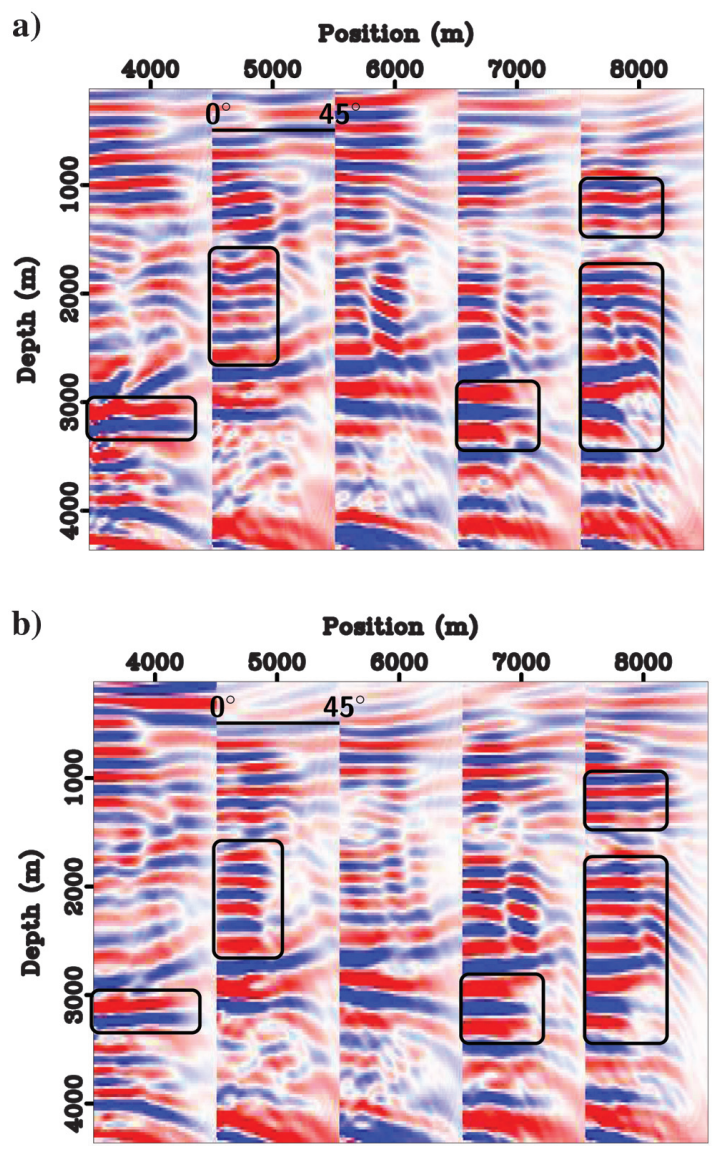

Figure 13. Angle gathers for (a) the initial and (b) the inverted model. The angle gathers are shown for multiple locations along the inline direction at $y=3 \mathrm{~km}$. 


\section{DISCUSSION}

The scattering potential of the selected parameterization reveals that the six orthorhombic parameters can be classified into three types depending on the strength of their influence on the data: one parameter that influences the data at all azimuths and all scattering angles $\left(v_{h 1}\right)$, two parameters that affect the data at all azimuths and certain scattering angles $\left(\epsilon_{1}\right.$ and $\left.\eta_{1}\right)$, and three parameters that affect the data at certain azimuths and certain scattering angles $\left(\epsilon_{d}, \delta_{3}\right.$, and $\left.\eta_{d}\right)$. These three classes correspond to three level of model symmetry, which are the isotropic, the VTI, and the orthorhombic model representation. Moreover, the scattering potential of these parameters is stationary (remains unchanged) as we move from a high symmetry model to a lower one, which gives us the opportunity to hierarchically update the three parameter classes.

We generally find that four parameters out of the six describing the orthorhombic medium may be recovered from wide-azimuth $\mathrm{P}$-wave surface seismic data with different resolution scales. Specifically, these parameters are $v_{h_{1}}, \epsilon_{1}, \epsilon_{d}$, and $\delta_{3}$. The velocity $v_{h 1}$ should be well-resolved. It has an isotropic radiation pattern and has the most influence on the data, among all other parameters. The parameter $\epsilon_{1}$ could be partially recovered. Indeed, this parameter affects vertically traveling waves; therefore, we may only recover its high-wavenumber information. It follows that a good initial $\epsilon_{1}$ is a requirement; otherwise, $\epsilon_{1}$ would just play the role of a "garbage" collector
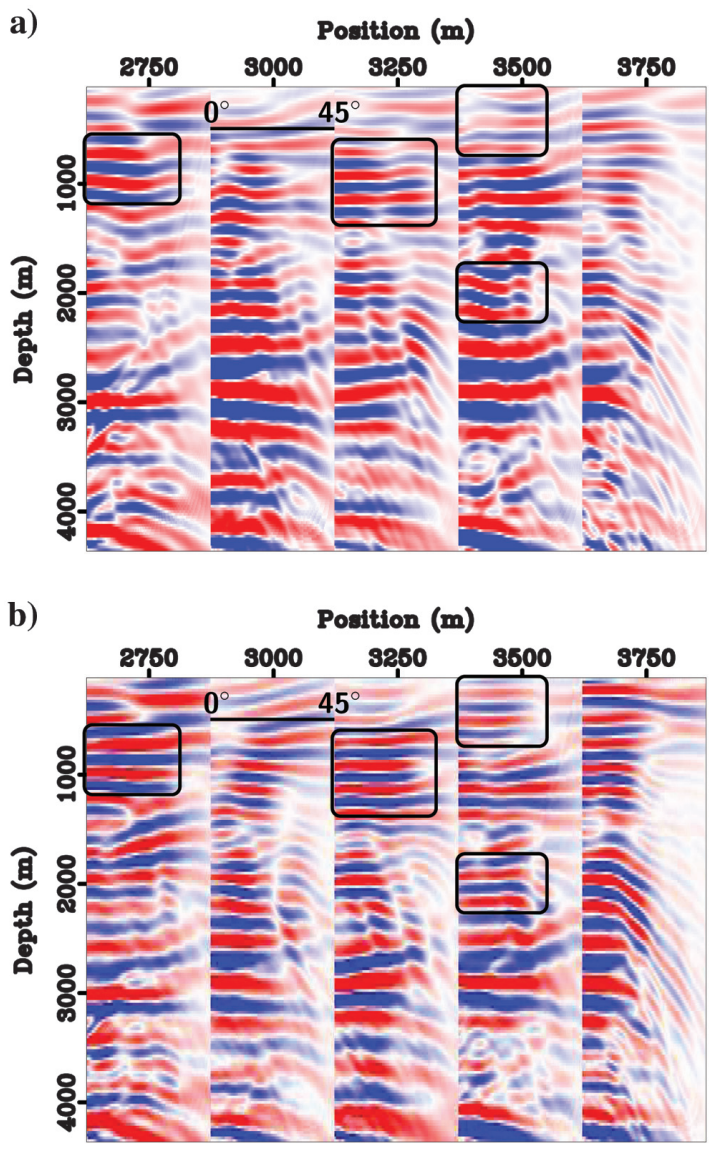

Figure 14. Angle gathers for (a) the initial and (b) the inverted model. The angle gathers are shown for multiple locations along the crossline direction at $x=6 \mathrm{~km}$. variable in absorbing the density and other data mismatch effects. Finally, smooth $\epsilon_{d}$ and $\delta_{3}$ could be recovered from wide-azimuth data. However, we find that $\epsilon_{d}$ could be better recovered than $\delta_{3}$ because it has stronger influence on large-offset data. The two remaining parameters $\eta_{1}$ and $\eta_{d}$ might be inverted as well; however, we may not trust their updates given their weak influence of the data and the potential leakage with other parameters. In a nutshell, we could use these initial conclusions to guide the real data applications, in terms of the parameters we should focus on, the resolution expected from each parameter, as well as the potential model regularization that could be applied during the inversion process.

The real data set used in this study is challenging for an orthorhombic model inversion due to the limited offsets especially in the crossline direction. This limitation affects the recovery of the three parameters describing the azimuthal anisotropy. Therefore, we excluded from the inversion $\delta_{3}$ and $\eta_{d}$ and we focused only on $\epsilon_{d}$, which we constrained to have a similar structure to $\epsilon_{1}$. Moreover, diving waves are needed to update the low-wavenumber content of the model. This is essential at the early stages of the inversion process (Bunks et al., 1995). Unfortunately, some of the diving waves are missing from the real data. This issue is compensated thanks to the availability of a good background model.

The inversion examples discussed here are based on a specific objective function, which is mostly sensitive to the phase information. Therefore, the resolution of the inverted model might be limited because of this choice. However, we find it to be reasonably practical for the acoustic approximation based modeling in which the dynamics are not accurate. Finally, the usage of more sophisticated objective functions should mitigate the requirement of accurate initial models.

\section{CONCLUSION}

Guided by the insights from the scattering potential analysis and the synthetic data inversion, we successfully applied a 3D acoustic orthorhombic waveform inversion to a field data set. The use of a specific parameterization that preserves the continuity of the scattering potential as we move from higher symmetry anisotropy to lower ones allowed us to propose a multistage model inversion strategy in which the isotropic, then the VTI, and finally the orthorhombic parameters are hierarchically updated. We have shown that P-wave surface seismic data have different sensitivity to the orthorhombic model parameters. The recovery of those depends on data quality and prior information. The synthetic data inversion using a variable density orthorhombic model illustrated the advantages and the limitations of the acoustic approximation. It also helped us explain the potential leakage of the elastic parameters into the acoustic ones. The case study from the North Sea demonstrated the challenges of inverting for the azimuthal anisotropy parameters especially when wide-azimuth information is limited. In this case, we have demonstrated the utility of using model constraints to steer the inverted model toward a more plausible subsurface representation. We have shown that the inverted model matches well data, generates synthetic data that match field data, and better flattens CIGs compared with the initial model.

\section{ACKNOWLEDGMENTS}

The authors would like to thank Statoil ASA and the Volve license partners ExxonMobil E\&P Norway AS and Bayerngas Norge 
AS, for the release of the Volve data. The views expressed in this paper are the views of the authors and do not necessarily reflect the views of Statoil ASA and the Volve field license partners. The authors would like to thank M. Houbiers from Statoil, who gave some helpful suggestions. For computer time, this research used the resources of the Supercomputing Laboratory at King Abdullah University of Science \& Technology (KAUST) in Thuwal, Saudi Arabia. We also acknowledge the valuable comments from three anonymous reviewers that significantly improved the quality of this manuscript. We are also grateful to S. Feki and B. Hadri from the KAUST Supercomputing Laboratory for many useful discussions. We also thank KAUST for financial support, J.-W. Oh for generating the synthetic data, and SWAG members at KAUST for the collaborative environment.

\section{APPENDIX A}

\section{THE PHASE OPERATOR}

The acoustic wave equation for anisotropic media satisfies a linear pseudodifferential equation of the form

$$
\left[\omega^{2}-\Phi(\mathbf{x}, \mathbf{k})\right] u(\mathbf{k}, \omega)=0
$$

where $u(\mathbf{k}, \omega)$ is the pressure wavefield, $\omega$ is the frequency, $\mathbf{k}$ is the wave vector, and $\Phi$ is the pseudodifferential phase operator. In the orthorhombic case, this yields the cubic polynomial (Song and Alkhalifah, 2013)

$$
-\Phi^{3}+a \Phi^{2}+b \Phi+c=0
$$

where the polynomial coefficients $a, b$, and $c$ are given below in terms of the parameterization considered in this paper:

$$
a=v_{h_{1}}^{2}\left(k_{x}^{2}+\left(1+2 \epsilon_{d}\right) k_{y}^{2}+\frac{1}{1+2 \epsilon_{1}} k_{z}^{2}\right),
$$

$$
\begin{gathered}
b=v_{h_{1}}^{4}\left(\left(\left(1+2 \delta_{3}\right)-\left(1+2 \epsilon_{d}\right)\right) k_{x}^{2} k_{y}^{2}-\frac{2 \eta_{1}}{\left(1+2 \eta_{1}\right)\left(1+2 \epsilon_{1}\right)} k_{x}^{2} k_{z}^{2}\right. \\
\left.-\frac{\left(1+2 \epsilon_{d}\right)\left(\left(1+2 \eta_{d}\right)\left(1+2 \eta_{1}\right)-1\right)}{\left(1+2 \eta_{d}\right)\left(1+2 \eta_{1}\right)\left(1+2 \epsilon_{1}\right)} k_{y}^{2} k_{z}^{2}\right),
\end{gathered}
$$

$$
\begin{aligned}
c & =v_{h_{1}}^{6}\left(-\frac{k_{x}^{2} k_{y}^{2} k_{z}^{2}}{\left(1+2 \epsilon_{1}\right)\left(1+2 \eta_{1}\right)}\left(\left(1+2 \eta_{1}\right)\left(1+2 \delta_{3}\right)\right.\right. \\
& \left.\left.-2 \sqrt{\frac{\left(1+2 \epsilon_{d}\right)\left(1+2 \delta_{3}\right)}{1+2 \eta_{d}}}+\left(1+2 \epsilon_{d}\right)\left(-2 \eta_{1}+\frac{1}{1+2 \eta_{d}}\right)\right)\right) .
\end{aligned}
$$

\section{APPENDIX B THE GRADIENTS}

Starting from the least-squares misfit function (equation 11) and using the adjoint state method, one can obtain the adjoint wavefield $\lambda$ (equation 14) as well as the gradients with respect to the model parameters $v_{h_{1}}, \epsilon_{1}, \epsilon_{d}, \eta_{1}, \eta_{d}$, and $\delta_{3}$ given below:

$$
\frac{\partial J_{d}}{\partial v_{h_{1}}}=\frac{2}{v_{h_{1}}^{3}} \int_{0}^{T} \lambda(\mathbf{x}, T-t)\left[\frac{\partial^{2} u}{\partial t^{2}}\right] d t,
$$

$$
\frac{\partial J_{d}}{\partial \epsilon_{1}}=\int_{0}^{T} \lambda(\mathbf{x}, T-t)\left[-2 S \frac{\partial^{2} u}{\partial z^{2}}+\frac{\partial S}{\partial \epsilon_{1}} \nabla_{E}^{2} u\right] d t
$$

$$
\frac{\partial J_{d}}{\partial \epsilon_{d}}=\int_{0}^{T} \lambda(\mathbf{x}, T-t)\left[2 S \frac{\partial^{2} u}{\partial y^{2}}+\frac{\partial S}{\partial \epsilon_{d}} \nabla_{E}^{2} u\right] d t
$$

$$
\frac{\partial J_{d}}{\partial \eta_{1}}=\int_{0}^{T} \lambda(\mathbf{x}, T-t)\left[\frac{\partial S}{\partial \eta_{1}} \nabla_{E}^{2} u\right] d t
$$

$$
\frac{\partial J_{d}}{\partial \eta_{d}}=\int_{0}^{T} \lambda(\mathbf{x}, T-t)\left[\frac{\partial S}{\partial \eta_{d}} \nabla_{E}^{2} u\right] d t,
$$

$$
\frac{\partial J_{d}}{\partial \delta_{3}}=\int_{0}^{T} \lambda(\mathbf{x}, T-t)\left[\frac{\partial S}{\partial \delta_{3}} \nabla_{E}^{2} u\right] d t,
$$

where the operators $S$ and $\nabla_{E}^{2}$ are given in equation 10 .

The partial derivatives of $S$ with respect to the model parameters are obtained using the approximate phase expression (equation 17)

$$
\begin{aligned}
\frac{\partial S}{\partial \epsilon_{d}}= & -\frac{2 n_{y}^{2}\left(1+2 n_{y}^{2}\left(n_{y}^{2}+n_{z}^{2}\right) \epsilon_{d}-2 n_{z}^{2} \epsilon_{1}-2\left(n_{x}^{2}+n_{y}^{2}\right) n_{z}^{2} \eta_{1}-2 n_{y}^{2} n_{z}^{2} \eta_{d}+2 n_{x}^{2} n_{y}^{2} \delta_{3}\right)}{A^{2}} \\
& +\frac{2 n_{y}^{2}\left(n_{y}^{2}+n_{z}^{2}\right)}{A},
\end{aligned}
$$

$$
\begin{gathered}
\frac{\partial S}{\partial \epsilon_{1}}=\frac{2 n_{z}^{2}\left(1+2 n_{y}^{2}\left(n_{y}^{2}+n_{z}^{2}\right) \epsilon_{d}-2 n_{z}^{2} \epsilon_{1}-2\left(n_{x}^{2}+n_{y}^{2}\right) n_{z}^{2} \eta_{1}-2 n_{y}^{2} n_{z}^{2} \eta_{d}+2 n_{x}^{2} n_{y}^{2} \delta_{3}\right)}{\left(1+2 \epsilon_{1}\right)^{2} A^{2}} \\
-\frac{2 n_{z}^{2}}{A}, \\
\frac{\partial S}{\partial \eta_{1}}=\frac{-2 n_{z}^{2}\left(n_{x}^{2}+n_{y}^{2}\right)}{A},
\end{gathered}
$$




$$
\frac{\partial S}{\partial \eta_{d}}=\frac{-2 n_{y}^{2} n_{z}^{2}}{A}
$$

$$
\frac{\partial S}{\partial \delta_{3}}=\frac{2 n_{x}^{2} n_{y}^{2}}{A}
$$

where

$$
A=\left(n_{x}^{2}+n_{y}^{2}\left(1+2 \epsilon_{d}+1 /\left(1+2 \epsilon_{1}\right) n_{z}^{2}\right)\right)^{2},
$$

where $n_{x}, n_{y}$, and $n_{z}$ are the components of the unit vector $\mathbf{n}$ of phase direction $\mathbf{n}=\mathbf{k} /|\mathbf{k}|$, approximated from the components of $\nabla u /|\nabla u|$ (Xu and Zhou, 2014).

\section{REFERENCES}

Albertin, U., P. Shen, A. Sekar, T. Johnsen, C. Wu, K. Nihei, and K. Bube, 2016, 3D orthorhombic elastic full-waveform inversion in the reflection domain from hydrophone data: 86th Annual International Meeting, SEG, Expanded Abstracts, 1094-1098.

Alkhalifah, T., 2000, An acoustic wave equation for anisotropic media: Geophysics, 65, 1239-1250, doi: 10.1190/1.1444815.

Alkhalifah, T., 2003, An acoustic wave equation for orthorhombic anisotropy: Geophysics, 68, 1169-1172, doi: 10.1190/1.1598109.

Alkhalifah, T., 2016, Research note: Insights into the data dependency on anisotropy: An inversion prospective: Geophysical Prospecting, 64, 505513, doi: $10.1111 / 1365-2478.12345$.

Alkhalifah, T., and A. Guitton, 2016, An optimal parameterization for full waveform inversion in anisotropic media: 78th Annual International Conference and Exhibition, EAGE, Extended Abstracts, We SRS2 05, doi: 10 3997/2214-

4609.201601192 .

Alkhalifah, T., N. Masmoudi, and J.-W. Oh, 2016, A recipe for practical fullwaveform inversion in orthorhombic anisotropy: The Leading Edge, 35 1076-1083, doi: 10.1190/tle35121076.1.

Alkhalifah, T., and R. E. Plessix, 2014, A recipe for practical full-waveform inversion in anisotropic media: An analytical parameter resolution study: Geophysics, 79, no. 3, R91-R101, doi: 10.1190/geo2013-0366.1.

Bakulin, A., V. Grechka, and I. Tsvankin, 2000, Estimation of fracture parameters from reflection seismic data. Part II: Fractured models with orthorhombic symmetry: Geophysics, 65, 1803-1817, doi: 10.1190/1 .1444864 .

Bunks, C., F. M. Saleck, S. Zaleski, and G. Chavent, 1995, Multiscale seismic waveform inversion: Geophysics, 60, 1457-1473, doi: 10.1190/1 .1443880 .

Cheadle, S. P., R. J. Brown, and D. C. Lawton, 1991, Orthorhombic anisotropy: A physical seismic modeling study: Geophysics, 56, 16031613, doi: 10.1190/1.1442971.

Cheng, J., and W. Kang, 2014, Simulating propagation of separated wave modes in general anisotropic media. Part I: qP-wave propagators: Geophysics, 79, no. 1, C1-C18, doi: 10.1190/geo2012-0504.1.

Choi, Y., and T. Alkhalifah, 2012, Application of multi-source waveform inversion to marine streamer data using the global correlation norm: Geophysical Prospecting, 60, 748-758, doi: 10.1111/j.1365-2478.2012.01079.x.

Clayton, R., and B. Engquist, 1977, Absorbing boundary conditions for acoustic and elastic wave equations: Bulletin of the Seismological Society of America, 67, 1529-1540.

Devaney, A. J., 1984, Geophysical diffraction tomography: IEEE Transactions on Geoscience and Remote Sensing, GE-22, 3-13, doi: 10.1109/ TGRS.1984.350573.

Diaz, E., and A. Guitton, 2011, Fast full waveform inversion with random shot decimation: 81st Annual International Meeting, SEG, Expanded Abstracts, 2804-2808.

Djebbi, R., and T. Alkhalifah, 2017, Frequency domain multi-parameter full waveform inversion for acoustic VTI media: 79th Annual International Conference and Exhibition, EAGE, Extended Abstracts, We B2 13, doi: 10.3997/2214-4609.201701231.

Djebbi, R., R.-É. Plessix, and T. Alkhalifah, 2017, Analysis of the traveltime sensitivity kernels for an acoustic transversely isotropic medium with a vertical axis of symmetry: Geophysical Prospecting, 65, 22-34, doi: $10.1111 / 1365-2478.12361$
Dussaud, E., W. W. Symes, P. Williamson, L. Lemaistre, P. Singer, B. Denel, and A. Cherrett, 2008, Computational strategies for reverse time migration: 78th Annual International Meeting, SEG, Expanded Abstracts, 2267-2271.

Fomel, S., L. Ying, and X. Song, 2013, Seismic wave extrapolation using low rank symbol approximation: Geophysical Prospecting, 61, 526-536, doi: $10.1111 / \mathrm{j} .1365-2478.2012 .01064 . x$.

Fowler, P. J., and R. King, 2011, Modeling and reverse time migration of orthorhombic pseudoacoustic P-waves: 81st Annual International Meeting, SEG, Expanded Abstracts, 190-195.

Fowler, P. J., and C. Lapilli, 2012, Generalized pseudospectral methods for orthorhombic modeling and reverse-time migration: 82nd Annual International Meeting, SEG, Expanded Abstracts, doi: 10.1190/segam20120708.1 .

Gallardo, L. A., and M. A. Meju, 2003, Characterization of heterogeneous near-surface materials by joint $2 \mathrm{D}$ inversion of DC resistivity and seismic data: Geophysical Research Letters, 30, 1658.

Gauthier, O., J. Virieux, and A. Tarantola, 1986, Two dimensional nonlinear inversion of seismic waveforms: Numerical results: Geophysics, 51, 13871403, doi: $10.1190 / 1.1442188$.

Gholami, Y., R. Brossier, S. Operto, A. Ribodetti, and J. Virieux, 2013, Which parameterization is suitable for acoustic vertical transverse isotropic media full waveform inversion? Part 1: Sensitivity and trade-off analysis: Geophysics, 78, no. 2, R81-R105, doi: 10.1190/geo2012-0204.1.

Guitton, A., 2012, Blocky regularization schemes for full-waveform inversion: Geophysical Prospecting, 60, 870-884, doi: 10.1111/j.1365-2478 $.2012 .01025 . x$

Guitton, A., and T. Alkhalifah, 2016, Full-waveform inversion in an anisotropic elastic earth-Can we isolate the role of density and shear wave velocity?: 78th Annual International Conference and Exhibition, EAGE, Extended Abstracts, We SRS2 06, doi: 10.3997/2214-4609 201601193.

He, W., and R.-É. Plessix, 2017, Analysis of different parameterizations of waveform inversion of compressional body waves in an elastic transverse isotropic earth with a vertical axis of symmetry: Geophysical Prospecting, 65, 1004-1024, doi: 10.1111/1365-2478.12452.

Hu, W., A. Abubakar, and T. M. Habashy, 2009, Joint electromagnetic and seismic inversion using structural constraints: Geophysics, $\mathbf{7 4}$, no. 6 , R99-R109, doi: 10.1190/1.3246586.

Jin, S., R. Madariaga, J. Virieux, and G. Lambaré, 1992, Two-dimensional asymptotic iterative elastic inversion: Geophysical Journal International, 108, 575-588, doi: 10.1111/j.1365-246X.1992.tb04637.x.

Kamath, N., and I. Tsvankin, 2016, Elastic full-waveform inversion for VTI media: Methodology and sensitivity analysis: Geophysics, 81, no. 2, C53-C68, doi: 10.1190/geo2014-0586.1.

Kamath, N., I. Tsvankin, and E. Diaz, 2017, Elastic full-waveform inversion for VTI media: A synthetic parameterization study: Geophysics, 82, no. 5, C163-C174, doi: 10.1190/geo2016-0375.1.

Kazei, V., and T. Alkhalifah, 2018, Waveform inversion for orthorhombic anisotropy with P-waves feasibility and resolution: Geophysical Journal International, 213, 963-982, doi: 10.1093/gji/ggy034.

Lailly, P., 1983, The seismic inverse problem as a sequence of before stack migrations: Conference on Inverse Scattering, Theory and Application, SIAM, Expanded Abstracts, 206-220.

Le, H., B. Biondi, R. G. Clapp, and S. A. Levin, 2015, Using a nonlinear acoustic wave equation for anisotropic inversion: 85th Annual International Meeting, SEG, Expanded Abstracts, 467-471.

Liu, Q., and J. Tromp, 2006, Finite-frequency kernels based on adjoint methods: Bulletin of the Seismological Society of America, 96, 2383-2397, doi: $10.1785 / 0120060041$

Masmoudi, N., and T. Alkhalifah, 2016a, A new parameterization for waveform inversion in acoustic orthorhombic media: Geophysics, 81, no. 4, R157-R171, doi: 10.1190/geo2015-0635.1.

Masmoudi, N., and T. Alkhalifah, 2016b, Scattering potential of acoustic orthorhombic parametrization: An inversion prospective: 78th Annual International Conference and Exhibition, EAGE, Extended Abstracts, We P1 11, doi: 10.3997/2214-4609.201601010.

Masmoudi, N., and T. Alkhalifah, 2017, Waveform inversion in acoustic orthorhombic media with a practical set of parameters: 87th Annual International Meeting, SEG, Expanded Abstracts, 1659-1665.

Masmoudi, N., I. Tsvankin, and T. Alkhalifah, 2016, Feasibility of high-resolution fracture characterization using waveform inversion: 78th Annual International Conference and Exhibition, EAGE, Extended Abstracts, We LHR5 13, doi: 10.3997/2214-4609.201600996.

Métivier, L., F. Bretaudeau, R. Brossier, S. Operto, and J. Virieux, 2014, Full waveform inversion and the truncated Newton method quantitative imaging of complex subsurface structures: Geophysical Prospecting, 62, 13531375, doi: 10.1111/1365-2478.12136.

Miller, D., M. Oristaglio, and G. Beylkin, 1987, A new slant on seismic imaging: Migration and integral geometry: Geophysics, 52, 943-964, doi: $10.1190 / 1.1442364$.

Mu, J., B. Tang, S. Xu, H. Zhou, and A. DeNosaquo, 2015, Quasi-P wave reverse time migration on seam dataset: 85th Annual International Meeting, SEG, Expanded Abstracts, 3991-3995. 
Oh, J-W, and T. Alkhalifah, 2016a, 3D elastic-orthorhombic anisotropic full-waveform inversion: Application to field OBC data: 86th Annual International Meeting, SEG, Expanded Abstracts, 1206-1210.

Oh, J.-W., and T. Alkhalifah, 2016b, Elastic orthorhombic anisotropic parameter inversion: An analysis of parameterization: Geophysics, 81, no. 6, C279-C293, doi: 10.1190/geo2015-0656.1.

Operto, S. R. Brossier, L. Combe, L. Métivier, A. Ribodetti, and J. Virieux, 2014, Computationally efficient three-dimensional acoustic finite-difference frequency-domain seismic modeling in vertical transversely isotropic media with sparse direct solver: Geophysics, 79, no. 5, T257-T275, doi: 10.1190/geo2013-0478.1.

Operto, S., Y. Gholami, V. Prieux, A. Ribodetti, R. Brossier, L. Métivier, and J. Virieux, 2013, A guided tour of multiparameter full-waveform inversion with multicomponent data: From theory to practice: The Leading Edge, 32, 1040-1054, doi: 10.1190/tle32091040.1.

Pan, W., K. A. Innanen, G. F. Margrave, M. C. Fehler, X. Fang, and J. Li, 2016, Estimation of elastic constants for HTI media using Gauss-Newton and full-Newton multiparameter full-waveform inversion: Geophysics, 81, no. 5, R275-R291, doi: 10.1190/geo2015-0594.1.

Panning, M. P., Y. Capdeville, and B. A. Romanowicz, 2009, Seismic waveform modeling in a 3-D earth using the Born approximation: Potentia shortcomings and a remedy: Geophysical Journal International, 177, 161-178, doi: 10.1111/j.1365-246X.2008.04050.x.

Pica, A., J. P. Diet, and A. Tarantola, 1990, Nonlinear inversion of seismic reflection data in a laterally invariant medium: Geophysics, 55, 284-292, doi: $10.1190 / 1.1442836$

Plessix, R.-E., 2006, A review of the adjoint-state method for computing the gradient of a functional with geophysical applications: Geophysical Journal International, 167, 495-503, doi: 10.1111/j.1365-246X.2006.02978.x.

Pratt, R. G., 1999, Seismic waveform inversion in the frequency domain. Part 1: Theory and verification in a physical scale model: Geophysics, 64, 888-901, doi: 10.1190/1.1444597.

Pratt, R. G., C. Shin, and G. J. Hick, 1998, Gauss-Newton and full Newton methods in frequency-space seismic waveform inversion: Geophysical Journal International, 133, 341-362, doi: 10.1046/j.1365-246X.1998.00498.x.

Sambridge, M. S., A. Tarantola, and B. L. N. Kennett, 1991, An alternative strategy for non-linear inversion of seismic waveforms: Geophysical Prospecting, 39, 723-736, doi: 10.1111/j.1365-2478.1991.tb00341.x.

Schoenberg, M., and K. Helbig, 1997, Orthorhombic media: Modeling elastic wave behavior in a vertically fractured earth: Geophysics, 62, 19541974, doi: 10.1190/1.1444297.

Schoenberg, M., and C. M. Sayers, 1995, Seismic anisotropy of fractured rock: Geophysics, 60, 204-211, doi: 10.1190/1.1443748.

Shin, C., S. Jang, and D. J. Min, 2001, Improved amplitude preservation for prestack depth migration by inverse scattering theory: Geophysical Prospecting, 49, 592-606, doi: 10.1046/j.1365-2478.2001.00279.x.

Song, X., and T. Alkhalifah, 2013, Modeling of pseudoacoustic P-waves in orthorhombic media with a low-rank approximation: Geophysics, 78 , no. 4, C33-C40, doi: 10.1190/geo2012-0144.1.
Szydlik, J., T. P. Smith, S. Way, L. Aamodt, and C. Friedrich, 2007, 3D PP/PS prestack depth migration on the Volve field: First Break, 25, 43-47.

Tarantola, A., 1984, Inversion of seismic reflection data in the acoustic approximation: Geophysics, 49, 1259-1266, doi: 10.1190/1.1441754.

Thomsen, L., 1986, Weak elastic anisotropy: Geophysics, 51, 1954-1966, doi: 10.1190/1.1442051.

Tsvankin, I., 1997, Anisotropic parameters and P-wave velocity for orthorhombic media: Geophysics, 62, 1292-1309, doi: 10.1190/1 .1444231 .

Tsvankin, I., and V. Grechka, 2011, Seismology of azimuthally anisotropic media and seismic fracture characterization: SEG.

Virieux, J., and S. Operto, 2009, An overview of full-waveform inversion in exploration geophysics: Geophysics, 74, no. 6, WCC1-WCC26, doi: 10 $.1190 / 1.3238367$.

Waheed, U., C. E. Yarman, and G. Flagg, 2014, An iterative fast sweeping based Eikonal solver for tilted orthorhombic media: 84th Annual International Meeting, SEG, Expanded Abstracts, 480-485.

Wang, H., and I. Tsvankin, 2016, Feasibility of waveform inversion in acoustic orthorhombic media: 86th Annual International Meeting, SEG, Expanded Abstracts, 311-316.

Warner, M., A. Ratcliffe, T. Nangoo, J. Morgan, A. Umpleby, N. Shah, V. Vinje, I. Stekl, L. Guasch, C. Win, G. Conroy, and A. Bertrand, 2013, Anisotropic 3D full-waveform inversion: Geophysics, 78, no. 2, R59-R80, doi: 10 $.1190 /$ geo2012-0338.1.

Wild, P., and S. Crampin, 1991, The range of effects of azimuthal isotropy and EDA anisotropy in sedimentary basins: Geophysical Journal International, 107, 513-529, doi: 10.1111/j.1365-246X.1991.tb01412.x.

Wu, R., and K. Aki, 1985, Scattering characteristics of elastic waves by an elastic heterogeneity: Geophysics, 50, 582-595, doi: 10.1190/1 .1441934 .

Wu, Z., and T. Alkhalifah, 2016, Waveform inversion for acoustic VTI media in frequency domain: 86th Annual International Meeting, SEG, Expanded Abstracts, 1184-1189.

Xu, S., B. Tang, J. Mu, and H. Zhou, 2015, Elliptic decomposition of quasi-P wave equation: 77th Annual International Conference and Exhibition, EAGE, Extended Abstracts, We P6 12, doi: 10.3997/2214-4609 .201413134

$\mathrm{Xu}, \mathrm{S}$., and H. Zhou, 2014, Accurate simulations of pure quasi-P-waves in complex anisotropic media: Geophysics, 79, no. 6, T341-T348, doi: 10 1190/geo2014-0242.1

Yang, P., J. Gao, and B. Wang, 2014, RTM using effective boundary saving: A staggered grid GPU implementation: Computers \& Geosciences, 68 , 64-72, doi: 10.1016/j.cageo.2014.04.004.

Zhang, Z., T. Alkhalifah, J.-W. Oh, and I. Tsvankin, 2017, Estimation of fracture parameters using elastic full-waveform inversion: 87th Annual International Meeting, SEG, Expanded Abstracts, 3272-3276. 